TRANSACTIONS OF THE

AMERICAN MATHEMATICAL SOCIETY

Volume 359, Number 6, June 2007, Pages 2501-2529

S 0002-9947(07)03942-6

Article electronically published on January 4, 2007

\title{
A GENERAL CONFORMAL GEOMETRIC REFLECTION PRINCIPLE
}

\author{
OLIVER ROTH
}

\begin{abstract}
We prove a generalization of the Schwarz-Carathéodory reflection principle for analytic maps $f$ from the unit disk into arbitrary Riemann surfaces equipped with a complete real analytic conformal Riemannian metric $\lambda(w)|d w|$. This yields a necessary and sufficient condition for $f$ to have an analytic continuation in terms of the pullback of the metric $\lambda(w)|d w|$ under the map $f$.
\end{abstract}

\section{Results}

Let $\mathbb{D}:=\{z \in \mathbb{C}:|z|<1\}$ denote the open unit disk in the complex plane $\mathbb{C}$. An open subarc of the unit circle $\partial \mathbb{D}:=\{z \in \mathbb{C}:|z|=1\}$ is an open connected proper subset of $\partial \mathbb{D}$.

Theorem 1.1. Let $I$ be an open subarc of $\partial \mathbb{D}$, let $R$ be a Riemann surface, which carries a complete real analytic conformal Riemannian metric $\lambda(w)|d w|$ and let $f: \mathbb{D} \rightarrow R$ be a non-constant analytic map. Then $f$ can be continued analytically across $I$ with $f(I) \subset R$ if and only if there exists a holomorphic function $h: I \rightarrow \mathbb{C}$ such that

$$
\lim _{z \rightarrow \xi} \frac{\lambda(f(z))\left|f^{\prime}(z)\right|}{\left|h^{\prime}(z)\right|}=1, \quad \xi \in I .
$$

Some remarks are in order.

Remarks 1.2. (a) Theorem 1.1 simultaneously generalizes the classical SchwarzCarathéodory reflection principle for holomorphic functions [20, 4] and a recent reflection principle due to Fournier and Ruscheweyh 8 . Indeed, if we choose the Riemann surface $R$ to be the complex plane $\mathbb{C}$ and the metric $\lambda(w)|d w|$ to be the euclidean metric, i.e., $\lambda(w)=1$, then Theorem 1.1 is simply a variant of the Schwarz-Carathéodory reflection principle; see Section 1.1] below. If $R$ is the unit disk $\mathbb{D}$ and the metric $\lambda(w)|d w|$ the hyperbolic metric, then Theorem 1.1] is (a slight extension of) the Fournier-Ruscheweyh reflection principle in [8]; $c f$. again Section 1.1 .

(b) Note that every Riemann surface carries a complete real analytic conformal Riemannian metric (with constant Gaussian curvature). This is a consequence of the Uniformization Theorem.

(c) The expression

$$
\lambda(f(z))\left|f^{\prime}(z)\right|
$$

Received by the editors January 20, 2005.

2000 Mathematics Subject Classification. Primary 30A99; Secondary 30F45.

(C)2007 American Mathematical Society Reverts to public domain 28 years from publication 
in (1.1) denotes the pullback of the metric $\lambda(w)|d w|$ under the map $f$. Hence $\lambda(f(z))\left|f^{\prime}(z)\right|$ is a well-defined function on $\mathbb{D}$; see Section 2, The phrase " $f: \mathbb{D} \rightarrow R$ can be continued analytically across $I$ with $f(I) \subset R$ " means there exists a domain $\Omega \supset \mathbb{D}$ with $I \subset \Omega$ and an analytic map $F: \Omega \rightarrow R$ such that $F=f$ in $\mathbb{D}$. This map $F$ is the unique analytic continuation of $f$ to $\Omega$. Also recall that a function $h: M \rightarrow \mathbb{C}$ is said to be holomorphic on a set $M \subseteq \mathbb{C}$ if it is defined and holomorphic in an open set $V \subseteq \mathbb{C}$ containing $M$.

(d) Just as with the Schwarz-Carathéodory reflection principle, Theorem 1.1 generalizes to non-constant analytic maps $f: D \rightarrow R$, where (i) $D$ is a domain in $\mathbb{C}$ with an open free analytic boundary arc $I$ or (ii) $D$ is a bordered Riemann surface with border $\Gamma$ and $I \subset \Gamma$. This will be discussed in Section 4.1

(e) In the special case of a conformal Riemannian metric with constant Gaussian curvature the actual method of analytic continuation of an analytic map $f: \mathbb{D} \rightarrow R$ satisfying the boundary condition (1.1) is closely related to the theory of complex Riccati differential equations; see Section 4.2 .

(f) As we shall see from the proof of Theorem 1.1 the assumption that $\lambda(w)|d w|$ is a complete conformal Riemannian metric on $R$ is only used in the 'if' part of Theorem 1.1 to show $f: \mathbb{D} \rightarrow \mathbb{R}$ has a continuous extension to the boundary arc $I$ with $f(I) \subset R ; c f$. Lemma 3.2 .

(g) The restriction in Theorem 1.1 that $\lambda(w)|d w|$ is a real analytic conformal Riemannian metric can be slightly relaxed. For the 'if' part of Theorem 1.1 it suffices to assume $\lambda(w)|d w|$ is a complete conformal Riemannian metric, which is real analytic in a neighborhood $U \subset R$ of $f(I)$; $c f$. Theorem 3.1 below. For the 'only if' part we need only that $\lambda(w)|d w|$ is real analytic in a neighborhood of $f(I)$.

The following examples indicate that the assumptions of Theorem 1.1 cannot be substantially weakened.

Example 1.3. Consider the analytic map $f$ from the unit disk $\mathbb{D}$ to the punctured plane $R=\mathbb{C} \backslash\{0\}$ given by

$$
f(z)=\sqrt{\frac{1+z}{1-z}}, \quad f(0)=1 .
$$

The function $\lambda(w)=|w|$ is real analytic on $R$, and therefore induces a real analytic conformal Riemannian metric $\lambda(w)|d w|$ on $R$, which is, however, not complete. Also,

$$
\frac{\lambda(f(z))\left|f^{\prime}(z)\right|}{\left|h^{\prime}(z)\right|}=1, \quad z \in \mathbb{D}
$$

where the function $h(z)=(1-z)^{-1}$ is analytic in a neighborhood of the open subarc $I:=\partial \mathbb{D} \backslash\{1\}$ of $\partial \mathbb{D}$. In particular, condition (1.1) is fulfilled. But clearly $f$ cannot be continued analytically or even only continuously into a neighborhood of $z=-1$. Hence we cannot drop the assumption that $\lambda(w)|d w|$ is a complete conformal Riemannian metric in the 'if' part of Theorem 1.1.

Example 1.4. Let $\Omega$ be a domain in the complex plane, which is bounded by a smooth but nowhere analytic Jordan curve 11 and let $f$ be a conformal map from $\mathbb{D}$ onto $\Omega$. An explicit example of such a weird conformal map is given by the gap

\footnotetext{
${ }^{1}$ I.e. the curve has a $C^{\infty}$ but nowhere analytic parametrization $\omega(\tau), 0 \leq \tau \leq 1$, with $\omega^{\prime}(\tau) \neq 0$.
} 
power series

$$
f(z)=1+2 z+\sum_{n=1}^{\infty} 2^{-n^{2}} z^{2^{n}}, \quad z \in \mathbb{D} .
$$

The Painlevé-Kellogg-Warschawski theorem (see [18, Chapter 3]) guarantees that $f$ and its inverse $f^{-1}$ extend to $C^{\infty}$ functions on $\overline{\mathbb{D}}$ and $\bar{\Omega}$, respectively. Moreover, $f^{\prime}(z)$ has a non-vanishing $C^{\infty}$-extension to $\overline{\mathbb{D}}$, which we also denote by $f^{\prime}(z)$. Thus the function

$$
\varphi(w):=\frac{1}{\left|f^{\prime}\left(f^{-1}(w)\right)\right|}, \quad w \in \bar{\Omega},
$$

is a $C^{\infty}$ function on $\bar{\Omega}$ and real analytic in $\Omega$. We can therefore apply the Whitney extension theorem [22], which gives us a $C^{\infty}$ extension $\tilde{\varphi}: \mathbb{C} \rightarrow \mathbb{R}$ of $\varphi$ that is real analytic on $\mathbb{C} \backslash \bar{\Omega}$. By compactness of $\bar{\Omega}$ and continuity of $\tilde{\varphi}$, we have $\tilde{\varphi} \geq \delta$ on some domain $G \supset \bar{\Omega}$ for some constant $\delta>0$. Now it is easy to modify $\tilde{\varphi}$ in order to obtain a $C^{\infty}$ extension $\lambda: \mathbb{C} \rightarrow \mathbb{R}$ of $\varphi: \bar{\Omega} \rightarrow \mathbb{R}$, which is real analytic on $G \backslash \partial \Omega$, and bounded below by the same $\delta>0$ on the entire complex plane. Consequently, $\lambda(w)|d w|$ is a complete smooth conformal Riemannian metric on $\mathbb{C}$ (see Lemma 2.2), which is real analytic in $G \backslash f(\partial \mathbb{D})$. Also,

$$
\lambda(f(z))\left|f^{\prime}(z)\right|=1, \quad z \in \mathbb{D},
$$

by construction, so condition (1.1) holds for every $\xi \in \partial \mathbb{D}$ with $h(z) \equiv z$. Since clearly $f$ has no analytic continuation across any point $\xi \in \partial \mathbb{D}$, we see that we have to assume $\lambda(w)|d w|$ is a real analytic conformal Riemannian metric at least in a neighborhood of $f(I)$ for the 'if' part of Theorem 1.1

Example 1.5. Let $\eta(t)$ be a $2 \pi$-periodic, positive and smooth function on the real axis, which is nowhere real analytic, and define $\lambda\left(e^{i t}\right):=\eta(t), t \leq 0<2 \pi$. As above we can extend $\lambda\left(e^{i t}\right)$ to a complete smooth conformal Riemannian metric $\lambda(w)|d w|$ on $\mathbb{C}$, which is real analytic in $\{z \in \mathbb{C}: r<|z|<1 / r\} \backslash \partial \mathbb{D}$ for some $r \in(0,1)$. Now consider the analytic map $f(z)=z$ from $\mathbb{D}$ into $R=\mathbb{C}$, and let $I$ be an open subarc of $\partial \mathbb{D}$. Then there cannot exist a holomorphic function $h: I \rightarrow \mathbb{C}$ such that

$$
\lim _{z \rightarrow \xi} \frac{\lambda(f(z))\left|f^{\prime}(z)\right|}{\left|h^{\prime}(z)\right|}=1, \quad \xi \in I,
$$

since this implies $\left|h^{\prime}\left(e^{i t}\right)\right|=\eta(t)$ for $e^{i t} \in I$, which is impossible as $\left|h^{\prime}\left(e^{i t}\right)\right|$ is real analytic, but $\eta(t)$ is not. Consequently, the conformal Riemannian metric $\lambda(w)|d w|$ has to be real analytic at least in a neighborhood of $f(I)$ also for the 'only if' part of Theorem 1.1

1.1. Reflection principles in euclidean, hyperbolic and spherical geometry. If we choose the Riemann surface $R$ to be the complex plane $\mathbb{C}$ and the conformal Riemannian metric $\lambda(w)|d w|$ to be the euclidean metric $\lambda_{\mathbb{C}}(w)|d w|:=$ $|d w|$, then Theorem 1.1 is simply the following variant of the classical SchwarzCarathéodory reflection principle.

Theorem A (Schwarz 20] and Carathéodory [4]). Let I be an open subarc of the unit circle and let $f: \mathbb{D} \rightarrow \mathbb{C}$ be a holomorphic function. Then $f$ can be continued analytically across $I$ with $f(I) \subset \mathbb{C}$ if and only if there exists a holomorphic function $h: I \rightarrow \mathbb{C}$ such that

$$
\lim _{z \rightarrow \xi} \frac{\left|f^{\prime}(z)\right|}{\left|h^{\prime}(z)\right|}=1, \quad \xi \in I .
$$


Note that the euclidean metric is a complete conformal Riemannian metric on the complex plane and has vanishing Gaussian curvature. We might call Theorem $\mathrm{A}$ the euclidean Schwarz-Carathéodory reflection principle.

Remark 1.6. The Schwarz reflection principle was first stated by H. A. Schwarz 20] in the following way. A holomorphic function $f: \mathbb{D} \rightarrow \mathbb{C}$, which has a continuous extension to an open subarc $I$ of the unit circle and maps $I$ onto a subset of the real axis, can be continued analytically across this subarc. Carathéodory observed in 4 there is no need to assume $f$ has a continuous extension to $I$. He showed that if all cluster points of the sequence $\left\{f\left(z_{n}\right)\right\}$ are real for any sequence $\left\{z_{n}\right\} \subset \mathbb{D}$ which converges to a point of $I$, then $f$ can be continued analytically across $I$. Equivalently, if

$$
\lim _{z \rightarrow \xi}|f(z)|=1, \quad \xi \in I,
$$

then $f$ can be continued analytically across $I$. Clearly, Theorem $\mathrm{A}$ is an immediate consequence of this Schwarz-Carathéodory reflection principle and was first stated in this form by Fournier and Ruscheweyh in 8 .

In [8] an analogous hyperbolic Schwarz-Carathéodory reflection principle was established, where the complex plane with euclidean geometry in Theorem $\mathrm{A}$ is replaced by the unit disk $\mathbb{D}$ with its natural hyperbolic geometry induced by the hyperbolic metric

$$
\lambda_{\mathbb{D}}(w)|d w|=\frac{|d w|}{1-|w|^{2}} .
$$

This metric is complete, real analytic and has constant Gaussian curvature -4 .

Theorem B (Fournier and Ruscheweyh [8). Let I be an open subarc of the unit circle and let $f: \mathbb{D} \rightarrow \mathbb{D}$ be a holomorphic function such that

$$
\sup _{z \in \mathbb{D}} \frac{\left|f^{\prime}(z)\right|}{1-|f(z)|^{2}}<\infty .
$$

Then $f$ can be continued analytically across $I$ with $f(I) \subset \mathbb{D}$ if and only if there exists a holomorphic function $h: I \rightarrow \mathbb{C}$ such that

$$
\lim _{z \rightarrow \xi} \frac{\left|f^{\prime}(z)\right|}{1-|f(z)|^{2}} \frac{1}{\left|h^{\prime}(z)\right|}=1, \quad \xi \in I .
$$

Remarks 1.7. (a) If $f: \mathbb{D} \rightarrow \mathbb{D}$ is a holomorphic function satisfying (1.3), then Lemma 3.2 below applied for $(R, \lambda)=\left(\mathbb{D}, \lambda_{\mathbb{D}}\right)$ shows $f$ extends continuously to $\overline{\mathbb{D}}$ with $f(\overline{\mathbb{D}}) \subseteq \mathbb{D}$. Thus the only-if part of Theorem 1.1 implies the only-if part of Theorem B so Theorem B is a (slightly weaker) special case of Theorem 1.1.

(b) The proof of the 'if' part of Theorem B given in 8 is quite involved. It is based on an ingenious application of the Schauder fixed point theorem to a fixed point equation arising from the Poisson-Jensen formula. This method, however, intermingles the problem of analytic continuation of a given analytic function $f$ : $\mathbb{D} \rightarrow \mathbb{D}$ satisfying (1.4) with the much more difficult problem of existence and uniqueness of such functions. For this reason it cannot be adapted to prove the more general Theorem 1.1, see [19]. Note that Theorem 1.1 provides a partial answer to the question raised in Remark (5) in [8, p. 355]. We also wish to point out that the proof of Theorem $B$ in $[8]$ is non-constructive, whereas the proof of the more general Theorem 1.1 given in Section 3.1 and Section 3.2 below is indeed constructive - in principle, at least. 
The next result gives a spherical Schwarz-Carathéodory reflection principle for meromorphic functions. It is just another special case of Theorem 1.1 and complements the aforementioned euclidean and hyperbolic reflection principles in a natural way.

Theorem 1.8. Let I be an open subarc of the unit circle and let $f$ be a meromorphic function in $\mathbb{D}$. Then $f$ can be continued meromorphically across $I$ if and only if there exists a holomorphic function $h: I \rightarrow \mathbb{C}$ such that

$$
\lim _{z \rightarrow \xi} \frac{\left|f^{\prime}(z)\right|}{1+|f(z)|^{2}} \frac{1}{\left|h^{\prime}(z)\right|}=1, \quad \xi \in I .
$$

Remark 1.9. For a meromorphic function $f$ in $\mathbb{D}$ the metric

$$
\lambda(z)|d z|:=\frac{\left|f^{\prime}(z)\right|}{1+|f(z)|^{2}}|d z|
$$

is just the pull-back of the spherical metric

$$
\lambda_{\mathbb{P}}(w)|d w|:=\frac{|d w|}{1+|w|^{2}}
$$

on the extended complex plane $\mathbb{P}$ by the analytic map $f: \mathbb{D} \rightarrow \mathbb{P} . \lambda_{\mathbb{P}}(w)|d w|$ is a complete real analytic conformal Riemannian metric on $\mathbb{P}$; its Gaussian curvature is +4 .

1.2. On Beurling's extension of the Riemann mapping theorem. In 1953 A. Beurling [3] proved the following important extension of the Riemann mapping theorem 2

Theorem C (Beurling). Let $\Phi(w)$ be a positive, continuous and bounded function defined for $|w|<\infty$ and let $w_{0}$ be a given point in the $w$-plane. Then there exists an analytic and univalent function $f: \mathbb{D} \rightarrow \mathbb{C}$ normalized by

$$
f(0)=w_{0}, \quad f^{\prime}(0)>0,
$$

and satisfying the non-linear boundary condition

$$
\lim _{|z| \rightarrow 1}\left(\left|f^{\prime}(z)\right|-\Phi(f(z))\right)=0 .
$$

Moreover, if $\log \Phi(w)$ is superharmonic, then there is exactly one such analytic and univalent function.

We call any normalized, analytic and univalent function $f: \mathbb{D} \rightarrow \mathbb{C}$ satisfying (1.7) a Beurling-Riemann mapping function (for $\Phi(w)$ ). Note that every BeurlingRiemann mapping function $f(z)$ is a Lipschitz map from the metric space $(\mathbb{D},|\cdot|)$ to the metric space $(\mathbb{C},|\cdot|)$, i.e.

$$
\left|f\left(z_{1}\right)-f\left(z_{2}\right)\right| \leq M \cdot\left|z_{1}-z_{2}\right|, \quad z_{1}, z_{2} \in \mathbb{D},
$$

with

$$
M:=\sup _{w \in \mathbb{C}} \Phi(w)<\infty .
$$

Hence $f(z)$ has a continuous extension to $\overline{\mathbb{D}}$, and $\partial f(\mathbb{D})$ is a closed curve, which

\footnotetext{
${ }^{2}$ We refer to [2, 7] 13] for recent generalizations of and variations on Beurling's theorem.
} 
admits the conformal parametrization

$$
\partial f(\mathbb{D}): \quad f\left(e^{i t}\right), \quad 0 \leq t \leq 2 \pi .
$$

Moreover, $\left|f^{\prime}(z)\right|$ has a continuous extension to $\overline{\mathbb{D}}$ with $\left|f^{\prime}(z)\right| \neq 0$.

If a Beurling-Riemann mapping function can be continued analytically across an open subarc $I$ of the unit circle, then the corresponding function $\Phi(f(z))$ will be real analytic on $I$ since $\Phi(f(z))=\left|f^{\prime}(z)\right|>0$ there. A partial converse is given by the following theorem, which is essentially another special case of Theorem 1.1.

Theorem 1.10. Let $\Phi(w)$ be a positive, continuous and bounded function defined for $|w|<\infty$, let $w_{0}$ be a given point in the $w$-plane, and let $f(z)$ be a BeurlingRiemann mapping function for $\Phi(w)$ normalized by (1.6). If $\Phi(w)$ is real analytic in a neighborhood of $f(I)$ for some open subarc I of the unit circle, then $f(z)$ has an analytic continuation across $I$.

In particular, if $\Phi(w)$ is real analytic in a neighborhood of $\partial f(\mathbb{D})$, then every Beurling-Riemann mapping function for $\Phi(w)$ has an analytic extension to some disk $|z|\langle\rho, \rho\rangle 1$. Hence, at least in this special case, the analytic properties of the function $\Phi(w)$ are reflected by the analytic properties of the corresponding mapping functions.

1.3. On a free boundary value problem for analytic functions. In [7] the following result was established.

Theorem D. Let $\Psi$ be a holomorphic function in a neighborhood of the closed unit disk $\overline{\mathbb{D}}$ with $\Psi([0,1]) \subset(0,1)$, and let $z_{1}, \ldots, z_{n}$ be prescribed points in $\mathbb{D}$. Then there exists a holomorphic function $F: \overline{\mathbb{D}} \rightarrow \mathbb{D}$ with the following properties:

(a) $F(0)=0$ and

$$
F^{\prime}(z)=h(z) \prod_{k=1}^{n} \frac{z-z_{k}}{1-\overline{z_{k}} z}
$$

with a holomorphic non-vanishing function $h: \overline{\mathbb{D}} \rightarrow \mathbb{C}$.

$$
\lim _{z \rightarrow \xi}\left(\left|F^{\prime}(z)\right|-\Psi\left(|F(z)|^{2}\right)\right)=0, \quad \xi \in \partial \mathbb{D} .
$$

In fact, every holomorphic function $F: \mathbb{D} \rightarrow \mathbb{D}$ satisfying the free boundary condition (1.8) has a holomorphic extension to a neighborhood of $\overline{\mathbb{D}}$.

Proposition 1.11. Let $\Psi$ be a holomorphic function in a neighborhood of the closed unit disk $\overline{\mathbb{D}}$ such that $\Psi:[0,1] \rightarrow(0,1)$. Then every holomorphic function $F: \mathbb{D} \rightarrow \mathbb{D}$ satisfying (1.8) can be continued holomorphically across $\partial \mathbb{D}$.

This is a fairly simple consequence of Theorem 1.1. see Section 3.4 below. We note that Proposition 1.11 does not follow from Theorem D, because, in general, there are many holomorphic functions $F: \mathbb{D} \rightarrow \mathbb{D}$ with prescribed critical points satisfying (1.8).

1.4. A heuristic argument and an extension of Theorem 1.1. We give a heuristic argument why Theorem 1.1 can be expected to be true.

Roughly speaking, the boundary condition (1.1) compares the two conformal Riemannian pseudo-metrics

$$
\lambda(f(z))\left|f^{\prime}(z)\right||d z|
$$


and

$$
\left|h^{\prime}(z)\right||d z|
$$

on the open subarc $I$. It says that on $I$ the conformal pseudo-metric $\lambda(f(z))\left|f^{\prime}(z)\right||d z|$ is comparable to the conformal pseudo-metric $\left|h^{\prime}(z)\right||d z|$, which has vanishing Gaussian curvature, i.e. is of euclidean type. So "locally" the conformal metric $\lambda(f(z))\left|f^{\prime}(z)\right||d z|$ is euclidean. Since the euclidean metric $\left|h^{\prime}(z)\right||d z|$ is defined in a neighborhood of $I$, it is therefore not too far-fetched to expect that $\lambda(f(z))\left|f^{\prime}(z)\right||d z|$ can be continued analytically across $I$.

This observation also suggests the following generalization of the 'if' part of Theorem 1.1.

Theorem 1.12. Let $I$ be an open subarc of $\partial \mathbb{D}$ and let $R$ be a Riemann surface, which carries a complete real analytic conformal Riemannian metric $\lambda(w)|d w|$. Then an analytic map $f: \mathbb{D} \rightarrow R$ can be continued analytically across $I$ with $f(I) \subset R$ if there exist a holomorphic function $h: I \rightarrow \mathbb{C}$ and a positive real analytic function $\mu: \mathbb{C} \rightarrow \mathbb{R}$ such that

$$
\lim _{z \rightarrow \xi} \frac{\lambda(f(z))\left|f^{\prime}(z)\right|}{\mu(h(z))\left|h^{\prime}(z)\right|}=1, \quad \xi \in I .
$$

Remarks 1.13. (a) Again, we need only assume that $\mu$ is defined (and positive and real analytic) in a neighborhood of $h(I)$.

(b) The boundary condition (1.9) shows that on the subarc $I$ the conformal pseudo-metric

$$
\lambda(f(z))\left|f^{\prime}(z)\right||d z|
$$

is comparable to the pullback

$$
\left(h^{*} \mu\right)(z)|d z|=\mu(h(z))\left|h^{\prime}(z)\right||d z|
$$

of the conformal Riemannian metric $\mu(w)|d w|$ on $R=\mathbb{C}$ under the holomorphic function $h: I \rightarrow \mathbb{C}$. So "locally" $\lambda(f(z))\left|f^{\prime}(z)\right||d z|$ agrees with the conformal pseudo-metric $\left(h^{*} \mu\right)(z)|d z|$, which by hypothesis is defined in a neighborhood of $I$.

(c) In order to compare two conformal Riemannian metrics $\lambda(w)|d w|$ and $\mu(w)|d w|$ on a Riemann surface $S$ it is natural to consider the quotient

$$
\frac{\lambda(w)|d w|}{\mu(w)|d w|}
$$

which is a well-defined function on $S$; see Section 2. This motivates the boundary conditions (1.1) and (1.9) in Theorems 1.1 and 1.12, which in this form can easily be extended to analytic maps $f$ from a (bordered) Riemann surface $S$ into a Riemann surface $R$; see Section 4.1 .

1.5. Contents. The plan of the present paper is as follows. In Section 2 we describe the main ingredients we need for our proofs: conformal metrics, Fatou's radial limit theorem and complex ODEs. The proofs themselves are given in Section 3. In a supplementary Section 4, we first state some extensions of Theorem 1.1 and Theorem 1.12 for analytic maps $f: D \rightarrow R$, where $D$ is a domain with a free analytic boundary arc or a bordered Riemann surface. We then discuss the method of analytic continuation used in the proof of Theorem 1.1. This is related to the theory of Riccati differential equations in the complex plane. 


\section{Preliminaries}

We begin with a resumé of results from geometric function theory and ODE theory, which will come into play in Section 3.

2.1. Conformal Riemannian metrics and pseudo-metrics. For completeness and in order to fix the notation we give a rapid review of conformal Riemannian metrics. Details may be found in [1, 11, 17.

Roughly, a conformal Riemannian metric on a Riemann surface $R$ is a Riemannian metric on $R$, which is compatible with the conformal structure of $R$.

Definition 2.1. A conformal pseudo-metric on a Riemann surface

$$
\left(R,\left\{\left(\varphi_{\alpha}, U_{\alpha}\right)\right\}_{\alpha \in A}\right)
$$

is a collection of functions $\left(\lambda_{\alpha}\right)_{\alpha \in A}$, where each $\lambda_{\alpha}$ is a real-valued non-negative function on $\varphi_{\alpha}\left(U_{\alpha}\right)$, with the property

$$
\lambda_{\alpha}(w)=\lambda_{\beta}\left(\varphi_{\alpha, \beta}(w)\right)\left|\varphi_{\alpha, \beta}^{\prime}(w)\right|, \quad w \in \varphi_{\alpha}\left(U_{\alpha} \cap U_{\beta}\right),
$$

whenever $U_{\alpha} \cap U_{\beta} \neq \emptyset$. Here, $\varphi_{\alpha}: U_{\alpha} \rightarrow \mathbb{C}, \alpha \in A$, are the local coordinates of the Riemann surface $R$ and $\varphi_{\alpha, \beta}:=\varphi_{\beta} \circ \varphi_{\alpha}^{-1}$ are the transition maps from $\varphi_{\alpha}\left(U_{\alpha} \cap U_{\beta}\right)$ onto $\varphi_{\beta}\left(U_{\alpha} \cap U_{\beta}\right)$.

Accordingly, a conformal pseudo-metric is denoted by $\lambda(w)|d w|$. For simplicity, we always assume $\lambda(w)|d w|$ is continuous on $R$, that means each $\lambda_{\alpha}$ is a continuous function on $\varphi_{\alpha}\left(U_{\alpha}\right)$. We call a conformal pseudo-metric $\lambda(w)|d w|$ real analytic in an open set $U \subset R$, if for each local coordinate $\varphi_{\alpha}$ the function $\lambda_{\alpha}$ is real analytic in $\varphi_{\alpha}\left(U_{\alpha} \cap U\right)$. Note that $\lambda_{\alpha}\left(\varphi_{\alpha}(p)\right)=0$ for one $\alpha \in A$ with $p \in U_{\alpha}$ implies $\lambda_{\beta}\left(\varphi_{\beta}(p)\right)=0$ for any local coordinate $\varphi_{\beta}$ whose domain $U_{\beta}$ contains the point $p$, so we can say a pseudo-metric vanishes at a point of the Riemann surface.

Definition 2.2. A nonvanishing continuous conformal pseudo-metric $\lambda(w)|d w|$ on a Riemann surface $R$ is called a conformal Riemannian metric or simply a conformal metric.

In general, it makes no sense to speak of the value of a conformal pseudometric $\lambda(w)|d w|$ at a given point $p \in R$. Note, however, if $R$ is a domain in the complex plane, then we often regard a pseudo-metric $\lambda(w)|d w|$ as a function $\lambda: R \rightarrow[0, \infty)$, i.e. we identify the metric with the function associated with the identity local coordinate. In this case we can speak of the value $\lambda(w)$ of the pseudometric $\lambda(w)|d w|$. On the other hand, if $\lambda(w)|d w|$ and $\mu(w)|d w|$ are two conformal pseudo-metrics on a Riemann surface $R$, which do not vanish simultaneously, then the transformation rule (2.1) for the functions $\lambda_{\alpha}$ and $\mu_{\alpha}$ shows that the values

$$
\frac{\lambda_{\alpha}\left(\varphi_{\alpha}(p)\right)}{\mu_{\alpha}\left(\varphi_{\alpha}(p)\right)}, \quad p \in U_{\alpha}
$$

do not depend on $\alpha$, so in this case the quotient of two conformal pseudo-metrics is a well-defined function on $R$ with values in $[0, \infty]$.

Every conformal metric $\lambda(w)|d w|$ induces a distance on the Riemann surface $R$ in the following way. Let

$$
\mathrm{d}_{\lambda}(p, q):=\inf \int_{\gamma} \lambda(w)|d w|,
$$


where the infimum is taken over all locally rectifiable paths $\gamma$ in $R$ which join the points $p \in R$ and $q \in R$. This defines a distance $\mathrm{d}_{\lambda}: R \times R \rightarrow \mathbb{R}$ and makes $R$ into a path-metric space in the sense of Gromov [10. We denote this metric space by $(R, \lambda)$. The topology of $(R, \lambda)$ is compatible with the given topology on $R$. We call a conformal metric $\lambda(w)|d w|$ on a Riemann surface $R$ complete, if the metric space $(R, \lambda)$ is complete. In this case, $(R, \lambda)$ has the following very important property.

Theorem 2.1 (Hopf-Rinow). If $R$ is a Riemann surface with a complete conformal Riemannian metric $\lambda(w)|d w|$, then every bounded set in the metric space $(R, \lambda)$ is precompact.

A quick proof of Theorem 2.1 can be found in [10, Chapter 1]. The following simple, but useful lemma has been used in Example 1.4.

Lemma 2.2. If $\lambda: \mathbb{C} \rightarrow \mathbb{R}$ is a positive continuous function, which is bounded away from 0 , i.e. $\lambda(w) \geq \delta$ on $\mathbb{C}$ for some constant $\delta>0$, then $\lambda(w)|d w|$ is a complete conformal metric on the complex plane $\mathbb{C}$.

Proof. Let $z_{0}$ and $z_{1}$ be two points in $\mathbb{C}$. Then

$$
\left|z_{1}-z_{0}\right|=\inf _{\gamma} \int_{\gamma}|d w|=\frac{1}{\delta} \inf _{\gamma} \int_{\gamma} \delta|d w| \leq \frac{1}{\delta} \inf _{\gamma} \int_{\gamma} \lambda(w)|d w|=\frac{1}{\delta} d_{\lambda}\left(z_{1}, z_{0}\right),
$$

where the infimum is taken over all (locally rectifiable) paths in $\mathbb{C}$ joining $z_{0}$ and $z_{1}$. Thus, if $\left\{z_{n}\right\}$ is a Cauchy sequence in $(\mathbb{C}, \lambda)$, then it is also a Cauchy sequence in $(\mathbb{C},|\cdot|)$. Since $(\mathbb{C},|\cdot|)$ is a complete metric space, $\left\{z_{n}\right\}$ converges in $(\mathbb{C},|\cdot|)$ to a point $z \in \mathbb{C}$, say. But the topologies of $(\mathbb{C},|\cdot|)$ and $(\mathbb{C}, \lambda)$ are compatible, so $\left\{z_{n}\right\}$ also converges in $(\mathbb{C}, \lambda)$ to $z \in \mathbb{C}$. Therefore, $(\mathbb{C}, \lambda)$ is a complete metric space.

Now let $f: S \rightarrow R$ be an analytic map between Riemann surfaces $S$ and $R$ and let $\lambda(w)|d w|$ be a conformal pseudo-metric on $R$. Then the pullback

$$
\left(f^{*} \lambda\right)(z)|d z|=\lambda(f(z))\left|f^{\prime}(z)\right||d z|
$$

of $\lambda(w)|d w|$ via $f$ is a conformal pseudo-metric on $S$. If $f$ is an analytic map from a domain $D \subseteq \mathbb{C}$ into a Riemann surface $R$ equipped with a conformal pseudo-metric $\lambda(w)|d w|$, then we always regard $\lambda(f(z))\left|f^{\prime}(z)\right||d z|$ as a continuous (non-negative) function on $D$, and we write $\lambda(f(z))\left|f^{\prime}(z)\right|$, for short; see Theorem 1.1 .

2.2. Fatou's theorem. We also repeatedly need the following half plane version of Fatou's well-known theorem on the boundary behaviour of bounded holomorphic functions.

Theorem 2.3. Let $S^{+}=\{x+i y:-r<x<r, 0<y<r\}$ for some $r>0$ and let $f: S^{+} \rightarrow \mathbb{C}$ be a bounded holomorphic function. Then the vertical limits

$$
\lim _{y \rightarrow 0+} f(x+i y)=: f(x)
$$

exist for almost every point $x \in(r, r)$, and the boundary function $f(x)$ belongs to $L^{\infty}(-r, r):=\{f:(-r, r) \rightarrow \mathbb{C}$ measurable and essentially bounded $\}$. 
2.3. Complex differential equations. Let $D$ and $G$ be two domains in the complex plane and $f: D \times G \rightarrow \mathbb{C}$ a holomorphic function. Then for every $z_{0} \in D$ and every $w_{0} \in G$ the initial value problem

$$
\begin{aligned}
& \varphi^{\prime}(z)=f(z, \varphi(z)) \\
& \varphi\left(z_{0}\right)=w_{0},
\end{aligned}
$$

has a unique holomorphic solution $\varphi(z)$ defined in some domain $U \subset D$ containing the initial point $z_{0}$. This is the statement of the classical existence and uniqueness theorem for complex ordinary differential equations. In this section we consider the initial value problem (2.2) when the initial point $z_{0}$ belongs to the boundary of $D$. The following lemma deals with the case that $D$ is a rectangle. This will suffice for our purposes.

Lemma 2.4. Let $G \subset \mathbb{C}$ be a domain, $S^{+}:=\{z \in \mathbb{C}:-r<\operatorname{Re} z<r, 0<$ $\operatorname{Im} z<r\}$ for some $r>0, f: S^{+} \times G \rightarrow \mathbb{C}$ a bounded holomorphic function, and $w_{0} \in G$. Then for almost every $z_{0} \in(-r, r)$ there exists a half-disk $U=\{z \in \mathbb{C}$ : $\left.\left|z-z_{0}\right|<\rho, \operatorname{Im} z>0\right\} \subset S^{+}$and a uniquely determined function $\varphi: \bar{U} \rightarrow \mathbb{C}$ with the following properties:

(i) $\varphi$ is continuous in $\bar{U}$ and $\overline{\varphi(U)} \subset G$;

(ii) $\varphi$ is holomorphic in $U$;

(iii) $\varphi^{\prime}(z)=f(z, \varphi(z))$ for all $z \in U$ and $\varphi\left(z_{0}\right)=w_{0}$.

Moreover,

(a) the vertical limit

$$
f(x, w):=\lim _{y \rightarrow 0+} f(x+i y, w)
$$

exists for a.e. $x \in I:=\left(z_{0}-\rho, z_{0}+\rho\right)$ and every $w \in G$;

(b) the function $x \mapsto f(x, w)$ is essentially bounded and measurable on I for each fixed $w \in G$, and the function $w \mapsto f(x, w)$ is holomorphic in $G$ for a.e. $x \in I$;

(c) the function $\varphi$ is absolutely continuous on $I$ and

(d) $\varphi^{\prime}(x)=f(x, \varphi(x))$ for a.e. $x \in I$.

Remark 2.5. The proof of Lemma 2.4 will show that the assertion of Lemma 2.4 holds for every point $z_{0} \in(-r, r)$ for which the limit

$$
\lim _{y \rightarrow 0+} f\left(z_{0}+i y, w\right)
$$

exists locally uniformly with respect to $w \in G$.

For the proof of Lemma 2.4 we need the following simple Fatou-type lemma.

Lemma 2.6. Let $G \subseteq \mathbb{C}$ be a domain,

$$
S^{+}:=\{z \in \mathbb{C}:-r<\operatorname{Re} z<r, 0<\operatorname{Im} z<r\}
$$

for some $r>0$ and $f: S^{+} \times G \rightarrow \mathbb{C}$ a bounded holomorphic function. Then there exists a set $J \subset(-r, r)$ of Lebesgue measure 0 such that the limit

$$
f(x, w):=\lim _{y \rightarrow 0+} f(x+i y, w)
$$

exists for every $x \in(-r, r) \backslash J$ and every $w \in G$. For fixed $x \in(-r, r) \backslash J$ this limit exists locally uniformly with respect to $w \in G$. 
Proof. Let $\left\{w_{k}\right\}$ be a sequence of points in $G$ which has an accumulation point $w_{0} \in G$. The functions $z \mapsto f\left(z, w_{k}\right)$ are holomorphic and bounded in $S^{+}$. Thus, by Fatou's Theorem 2.3 there exists a set $J \subseteq(-r, r)$ of Lebesgue measure 0 s.t. the limits

$$
\lim _{y \rightarrow 0+} f\left(x+i y, w_{k}\right)
$$

exist for all $x \in(-r, r) \backslash J$ and every $k=1,2, \ldots$.

Now let $\left\{y_{n}\right\}$ be a sequence of positive real numbers with limit 0 and fix $x \in$ $(-r, r) \backslash J$. Then the functions $w \mapsto f\left(x+i y_{n}, w\right)$ form a normal family of holomorphic functions in $G$ such that

$$
\lim _{n \rightarrow \infty} f\left(x+i y_{n}, w_{k}\right)
$$

exists for every $k=1,2, \ldots$. By Vitali's theorem the limit

$$
\lim _{n \rightarrow \infty} f\left(x+i y_{n}, w\right)
$$

exists locally uniformly with respect to $w \in G$. It follows that the limit

$$
\lim _{y \rightarrow 0+} f(x+i y, w)
$$

exists locally uniformly with respect to $w \in G$.

We are now in a position to prove Lemma 2.4.

Proof of Lemma 2.4. Let $z_{0} \in \mathbb{R} \cap \partial U$ be a point such that the limit

$$
f\left(z_{0}, w\right):=\lim _{y \rightarrow 0+} f\left(z_{0}+i y, w\right)
$$

exists locally uniformly for $w \in G$; cf. Lemma 2.6. We can assume $z_{0}=0$ and $w_{0}=0$.

Choose $R>0$ with

$$
V:=\{w \in \mathbb{C}:|w|<R\}, \quad \bar{V} \subset G,
$$

let

$$
c:=\sup \left\{|f(z, w)|: z \in S^{+}, w \in G\right\}<\infty
$$

and let

$$
U:=\{z \in \mathbb{C}:|z|<\rho, \operatorname{Im} z>0\} \quad \text { where } \rho:=\min \left\{r, \frac{R}{4 c}\right\} .
$$

We fix $a \in(0, \rho)$ and denote the segment $\{i t: t \in[0, a]\}$ by $[0, i a]$. Since the function $f:[0, i a] \times G \rightarrow \mathbb{C}$ is continuous and $w \mapsto f(y, w)$ is analytic in $G$ for each fixed $y \in[0, i a]$, the initial value problem

$$
\begin{aligned}
\frac{d}{d y} \varphi_{1}(y) & =i f\left(i y, \varphi_{1}(y)\right), \quad 0 \leq y \leq a, \\
\varphi_{1}(0) & =0,
\end{aligned}
$$

has a unique solution $\varphi_{1}$ on the interval $0 \leq y \leq a$ with $\varphi_{1}(a) \in G$ by the standard existence and uniqueness theorem; see, for instance, [5, p. 10].

We now consider the "complex" initial value problem

$$
\begin{aligned}
\varphi^{\prime}(z) & =f(z, \varphi(z)), \quad z \in U, \\
\varphi(i a) & =\varphi_{1}(a) .
\end{aligned}
$$


We claim (2.7) has a unique analytic solution $\varphi: U \rightarrow V$. This can be seen as follows. Let $U^{*} \subset U$ be a domain with $i a \in U^{*}$ such that $\varphi$ is a solution to (2.7) on $U^{*}$ and $\varphi\left(U^{*}\right) \subset V$. Then, by (2.4) and (2.5),

$$
|\varphi(z)-\varphi(i a)| \leq c|z-i a|<c(\rho+\rho) \leq \frac{R}{2} .
$$

In view of (2.7) and (2.6) we have $|\varphi(i a)|=\left|\varphi_{1}(a)\right| \leq c a<R / 4$. Thus

$$
|\varphi(z)| \leq \frac{3 R}{4} \quad \text { for all } z \in U^{*}
$$

Consequently, the solution $\varphi: U^{*} \rightarrow V$ has an analytic continuation to all of $U$. Since $\varphi(U) \subset V$, it follows from (2.7) that $\left|\varphi^{\prime}(z)\right| \leq c$ for all $z \in U$. Therefore, $\varphi$ has a continuous extension to $\bar{U}$, which we also denote by $\varphi$ for simplicity.

We next show $\varphi(0)=0$. Observe that $\varphi_{2}(y):=\varphi(i y), 0<y \leq a$, is a solution of the initial value problem

$$
\begin{aligned}
\frac{d}{d y} \varphi_{2}(y) & =i f\left(i y, \varphi_{2}(y)\right) \\
\varphi_{2}(a) & =\varphi_{1}(a)
\end{aligned}
$$

so $\varphi_{2} \equiv \varphi_{1}$, i.e., $\varphi(i y)=\varphi_{1}(y)$ for $0<y \leq a$. In particular, $\varphi(0)=\varphi_{1}(0)=0$. Note that if $\tilde{\varphi}: \bar{U} \rightarrow G$ is another function with the properties (i)-(iii), then the same argument shows $\tilde{\varphi}=\varphi$ on $[0, i a]$ and hence on $U$.

Now we prove (a)-(d). The statements (a) and (b) follow immediately from Lemma 2.6 and Fatou's Theorem 2.3. In particular, the function $x \mapsto f(x, \varphi(x))$ is a Lebesgue-integrable function on the interval $I$ and

$$
\lim _{y \rightarrow 0+} f(x+i y, \varphi(x+i y))=f(x, \varphi(x)) \quad \text { for a.e. } x \in I .
$$

In view of

$$
\varphi(x+i y)-\varphi\left(x_{0}+i y\right)=\int_{x_{0}}^{x} f(t+i y, \varphi(t+i y)) d t
$$

for fixed $x, x_{0} \in I$ and all $y>0$ sufficiently small, we thus conclude

$$
\varphi(x)-\varphi\left(x_{0}\right)=\int_{x_{0}}^{x} f(t, \varphi(t)) d t
$$

by the dominated convergence theorem. This proves (c) and (d) and completes the proof of Lemma 2.4 .

\section{Proofs}

We are now prepared to prove Theorem 1.1, Theorem 1.10, Proposition 1.11 and Theorem 1.12. But before we do so, we introduce the following notation. The open disk of radius $r>0$ about $z_{0} \in \mathbb{C}$ will be denoted by $K_{r}\left(z_{0}\right)$. Also, $K_{r}^{+}(0)=$ $K_{r}(0) \cap \mathbb{H}$ is the upper half of the disk $K_{r}(0)$, where $\mathbb{H}=\{z \in \mathbb{C}: \operatorname{Im} z>0\}$ is the upper half plane, and $K_{r}^{-}(0)=\left\{z \in K_{r}(0): \operatorname{Im} z<0\right\}$ is the lower half of the disk $K_{r}(0)$. Finally, for complex numbers $a$ and $b$ we denote by $(a, b)$ the open segment joining $a$ and $b$, and by $[a, b]$ the closed segment between $a$ and $b$. 
3.1. Proof of the 'if' part of Theorem 1.1, The 'if' part of Theorem 1.1 follows from

Theorem 3.1. Let $I$ be an open subarc of $\partial \mathbb{D}$, let $R$ be a Riemann surface, which carries a conformal metric $\lambda(w)|d w|$, let $f: \mathbb{D} \rightarrow R$ be an analytic map, and let $h: I \rightarrow \mathbb{C}$ be a holomorphic function.

(a) If $\lambda(w)|d w|$ is complete and

$$
\lim _{z \rightarrow \xi} \frac{\lambda(f(z))\left|f^{\prime}(z)\right|}{\left|h^{\prime}(z)\right|}=1, \quad \xi \in I,
$$

then $f$ has a continuous extension $f: \mathbb{D} \cup I \rightarrow R$.

(b) If $f$ has a continuous extension $f: \mathbb{D} \cup I \rightarrow R, \lambda(w)|d w|$ is real analytic in a neighborhood of $f(I)$, and (3.1) holds, then $f$ can be continued analytically across $I$.

Theorem 3.1 will be established in several steps. We first prove the following extension of Lemma 2.1 in 8 .

Lemma 3.2. Let $\Omega$ be a bounded domain in the complex plane and assume there exists a constant $K=K(\Omega)>0$ such that for any pair of points $z_{1}, z_{2} \in \Omega$ there is an arc $\gamma$ in $\Omega$ joining $z_{1}$ and $z_{2}$ of euclidean length $l(\gamma) \leq K\left|z_{1}-z_{2}\right|$. Also, let $R$ be a Riemann surface equipped with a complete conformal metric $\lambda(w)|d w|$. Then every analytic map $f: \Omega \rightarrow R$ with

$$
\lambda(f(z))\left|f^{\prime}(z)\right| \leq M, \quad z \in \Omega,
$$

for some constant $M>0$, extends continuously to a map $f: \bar{\Omega} \rightarrow R$.

Proof. Take two points $z_{1}$ and $z_{2}$ in $\Omega$ and let $\gamma$ be an arc in $\Omega$ joining $z_{1}$ and $z_{2}$ of euclidean length $l(\gamma) \leq K\left|z_{1}-z_{2}\right|$. Then $f \circ \gamma$ is a (locally rectifiable) curve in $R$ joining $f\left(z_{1}\right)$ and $f\left(z_{2}\right)$, and we obtain the following uniform bound for the $\lambda$-distance $d_{\lambda}\left(f\left(z_{1}\right), f\left(z_{2}\right)\right)$ of $f\left(z_{1}\right)$ and $f\left(z_{2}\right)$ :

$d_{\lambda}\left(f\left(z_{1}\right), f\left(z_{2}\right)\right) \leq \int_{f \circ \gamma} \lambda(w)|d w|=\int_{\gamma} \lambda(f(z))\left|f^{\prime}(z)\right||d z| \leq M \cdot l(\gamma) \leq M \cdot K \cdot\left|z_{1}-z_{2}\right|$.

Hence $f:(\Omega,|\cdot|) \rightarrow(R, \lambda)$ is a Lipschitz map. Since $(R, \lambda)$ is complete, $f$ can be extended to a Lipschitz map from $(\bar{\Omega},|\cdot|)$ to $(R, \lambda)$. Note that $f(\Omega) \subset R$ is precompact in view of the Hopf-Rinow Theorem 2.1

Remark 3.3. Every bounded uniform domain (see [9, p. 37]) and hence every bounded quasidisk satisfies the hypotheses of Lemma 3.2. For the special case that $\Omega$ is a Jordan domain with Dini-smooth boundary (see [18, Chapter 3]) and $(R, \lambda)=\left(\mathbb{D}, \lambda_{\mathbb{D}}\right)$, Lemma 3.2 was proven in 8 by a completely different method.

Lemma 3.4. Let $I=(-\delta, \delta) \subseteq \mathbb{R}$ be an open interval with $\delta>0$, let $h: I \rightarrow \mathbb{C}$ be a holomorphic function and let $f: K_{\delta}^{+}(0) \cup I \rightarrow \mathbb{C}$ be a continuous function, which is holomorphic in $K_{\delta}^{+}(0)$. Finally, let $\mu$ be a positive real analytic function in a neighborhood of $f(I)$. If

$$
\lim _{z \rightarrow \xi} \frac{\mu(f(z))\left|f^{\prime}(z)\right|}{\left|h^{\prime}(z)\right|}=1, \quad \xi \in I,
$$

then $f$ has an analytic continuation $F$ to the disk $K_{r}(0)$ for some $r \in(0, \delta)$. 
Proof. We begin by translating the boundary condition (3.3) into a non-linear ordinary differential equation for the boundary function $f: I \rightarrow \mathbb{C}$.

Choose a number $r \in(0, \delta)$ such that $h(z)$ is holomorphic in a neighborhood of the square $S:=(-r, r) \times(-r, r) \subset K_{\delta}(0)$ and such that

$$
\frac{1}{2} \leq \frac{\mu(f(z))\left|f^{\prime}(z)\right|}{\left|h^{\prime}(z)\right|} \leq 2
$$

in $S \cap \mathbb{H}$. We may assume $\mu(f(z))$ is a well-defined continuous function in the upper half $S^{+}:=(-r, r) \times(0, r)$ of $S$ and bounded below by a positive constant. Since $\left|h^{\prime}(z)\right|$ is bounded above in $S$, we see from (3.4) that $f^{\prime}(z)$ and $h^{\prime}(z) / f^{\prime}(z)$ are bounded holomorphic functions in $S \cap \mathbb{H}$. By Fatou's theorem (Theorem 2.3), the vertical limits

$$
f^{\prime}(x):=\lim _{y \rightarrow 0+} f^{\prime}(x+i y), \quad \frac{h^{\prime}(x)}{f^{\prime}(x)}=\lim _{y \rightarrow 0+} \frac{h^{\prime}(x+i y)}{f^{\prime}(x+i y)}
$$

exist for almost every $x \in(-r, r)$. Moreover, $f^{\prime}(x)$ and $h^{\prime}(x) / f^{\prime}(x)$ are $L^{\infty_{-}}$ functions on the interval $(-r, r)$, and $f:(-r, r) \rightarrow \mathbb{C}$ is absolutely continuous with

$$
\frac{d}{d x} f(x)=f^{\prime}(x)
$$

almost everywhere on $(-r, r)$. Thus the boundary condition (3.3) can be written in the form

$$
\left|f^{\prime}(x)\right|=\frac{\left|h^{\prime}(x)\right|}{\mu(f(x))}
$$

or

$$
\frac{d}{d x} \overline{f(x)}=\frac{h^{\prime}(x)}{f^{\prime}(x)} \overline{h^{\prime}(x)} \frac{1}{\mu(f(x))^{2}}
$$

for almost every $x \in(-r, r)$. We note that the functions

$$
y \mapsto \frac{h^{\prime}(x+i y)}{f^{\prime}(x+i y)}
$$

are continuous on the interval $[0, r)$ for almost every $x \in(-r, r)$. Without loss of generality, we may assume the limits in (3.5) exist for $x=0$, so

$$
y \mapsto \frac{h^{\prime}(i y)}{f^{\prime}(i y)}
$$

is continuous on $[0, r)$. Finally, since $1 / \mu^{2}$ is real analytic in a neighborhood of $w_{0}=\xi_{0}+i \eta_{0}:=f(0)$ with $\xi_{0}, \eta_{0} \in \mathbb{R}$, it has a holomorphic extension $\Phi$ to the set $K_{\rho}\left(\xi_{0}\right) \times K_{\rho}\left(\eta_{0}\right) \subset \mathbb{C}^{2}$ for some $\rho>0$, and

$$
\Phi(\xi, \eta)=\frac{1}{\mu(\xi+i \eta)^{2}}
$$

for $\xi \in K_{\rho}\left(\xi_{0}\right) \cap \mathbb{R}$ and $\eta \in K_{\rho}\left(\eta_{0}\right) \cap \mathbb{R}$. Now our boundary condition takes the form

$$
\frac{d}{d x} \overline{f(x)}=\frac{h^{\prime}(x)}{f^{\prime}(x)} \overline{h^{\prime}(x)} \Phi\left(\frac{1}{2}(f(x)+\overline{f(x)}), \frac{1}{2 i}(f(x)-\overline{f(x)})\right)
$$

for almost every $x \in(-r, r)$, if $r>0$ is sufficiently small. 
Next, let us assume for a moment $f$ does have an analytic continuation $F$ to the square $S$. We may suppose

$$
\frac{1}{2}(F(z)+\overline{F(\bar{z})}) \in K_{\rho}\left(\xi_{0}\right), \quad \frac{1}{2 i}(F(z)-\overline{F(\bar{z})}) \in K_{\rho}\left(\eta_{0}\right), \quad z \in S,
$$

and $F^{\prime}(z) / h^{\prime}(z) \neq 0$ in $S$. Then (3.3) yields

or

$$
f^{\prime}(z) \overline{F^{\prime}(z)}=h^{\prime}(z) \overline{h^{\prime}(z)} \frac{1}{\mu(f(z))^{2}}, \quad z \in(-r, r),
$$

$f^{\prime}(z) \overline{F^{\prime}(\bar{z})}=h^{\prime}(z) \overline{h^{\prime}(\bar{z})} \Phi\left(\frac{1}{2}(f(z)+\overline{F(\bar{z})}), \frac{1}{2 i}(f(z)-\overline{F(\bar{z})})\right), \quad z \in(-r, r)$.

By the identity principle, the last identity holds for all $z \in S$. In other words, the function $g(z):=\overline{F(\bar{z})}$ is a solution of the complex ordinary differential equation

$$
g^{\prime}(z)=\frac{h^{\prime}(z)}{f^{\prime}(z)} \overline{h^{\prime}(\bar{z})} \Phi\left(\frac{1}{2}(f(z)+g(z)), \frac{1}{2 i}(f(z)-g(z))\right)
$$

in $S$, and

$$
g(z)=\overline{f(z)}, \quad z \in(-r, r) .
$$

This reasoning is reversible. If we can find a continuous function $g$ on $S^{+}=$ $(-r, r) \times[0, r)$, which is a holomorphic solution of the differential equation (3.7) in $(-r, r) \times(0, r)$ and satisfies (3.8), then the function

$$
F(z):= \begin{cases}f(z), & z \in(-r, r) \times(0, r), \\ \overline{g(\bar{z})}, & z \in(-r, r) \times(-r, 0],\end{cases}
$$

is continuous on $S$ and analytic in $S \backslash(-r, r)$. Thus, by the continuity principle (6, p. 13]), $F$ is the analytic continuation of $f$ across $(-r, r)$ to the square $S$ which we have been looking for.

We are now going to construct the function $g: S^{+} \rightarrow \mathbb{C}$. Note that $g$ must be a solution of the ordinary differential equation (3.7) and $g(0)=\overline{f(0)}$. Thus we are led to consider the complex differential equation

$$
\varphi^{\prime}(z)=\frac{h^{\prime}(z)}{f^{\prime}(z)} \overline{h^{\prime}(\bar{z})} \Phi\left(\frac{1}{2}(f(z)+\varphi(z)), \frac{1}{2 i}(f(z)-\varphi(z))\right)
$$

with initial condition

$$
\varphi(0)=\overline{f(0)}
$$

on the boundary $0 \in \partial S^{+}$. For sufficiently small $r>0$ the right-hand side of the differential equation (3.9),

$$
(z, w) \mapsto \tilde{f}(z, w):=\frac{h^{\prime}(z)}{f^{\prime}(z)} \overline{h^{\prime}(\bar{z})} \Phi\left(\frac{1}{2}(f(z)+w), \frac{1}{2 i}(f(z)-w)\right),
$$

is defined for $(z, w) \in S^{+} \times V$, where $V \subseteq \mathbb{C}$ is a disk about $\overline{f(0)}$. Moreover, the function $\tilde{f}: S^{+} \times V \rightarrow \mathbb{C}$ is a bounded holomorphic function and the limit

$$
\lim _{y \rightarrow 0+} \tilde{f}(i y, w)
$$

exists by construction.

Using Lemma 2.4 and Remark 2.5, and shrinking $r>0$ further, if necessary, we obtain a unique continuous solution $g:(-r, r) \times[0, r) \rightarrow V$ of the initial value 
problem (3.9)-(3.10). This solution is analytic in $S^{+}$, and the function $x \mapsto g(x)$ is absolutely continuous on $(-r, r)$ with

$$
g^{\prime}(x)=\tilde{f}(x, g(x)) \quad \text { for a.e. } x \in(-r, r) .
$$

But the function $x \mapsto \overline{f(x)}$ is an absolutely continuous solution of the same differential equation; see (3.6), and $g(0)=\overline{f(0)}$. Now

$$
(z, w) \mapsto \Phi\left(\frac{1}{2}(f(z)+w), \frac{1}{2 i}(f(z)-w)\right)
$$

is continuous on $((-r, r) \times[0, r)) \times V$ and continuously differentiable there with respect to $w$. In particular, $\tilde{f}$ is locally Lipschitz with respect to $w$ on $(-r, r) \times V$. Thus the standard uniqueness theorem for solutions of ODEs implies $g(x)=\overline{f(x)}$ for all $x \in(-r, r)$. This completes the proof of Lemma 3.4 .

The following lemma generalizes Lemma 3.4 to analytic maps $f: \mathbb{H} \rightarrow R$, where $R$ is a Riemann surface, which carries a conformal metric $\lambda(w)|d w|$.

Lemma 3.5. Let $I=(-\delta, \delta)$ be an open interval with $\delta>0$, let $R$ be a Riemann surface, let $h: I \rightarrow \mathbb{C}$ be a holomorphic function and let $f: \mathbb{H} \cup(-\delta, \delta) \rightarrow R$ be a continuous map, which is analytic in $\mathbb{H}$. Finally, let $\lambda(w)|d w|$ be a conformal metric on $R$, which is real analytic in a neighborhood of $f(I)$. If

$$
\lim _{z \rightarrow \xi} \frac{\lambda(f(z))\left|f^{\prime}(z)\right|}{\left|h^{\prime}(z)\right|}=1, \quad \xi \in I,
$$

then $f$ has an analytic continuation $F: \mathbb{H} \cup K_{r}(0) \rightarrow R$ to the disk $K_{r}(0)$ for some $r \in(0, \delta)$.

Proof. Let $X$ be the universal covering surface of $R$ and let $\pi: X \rightarrow R$ be an analytic universal cover projection. Thus

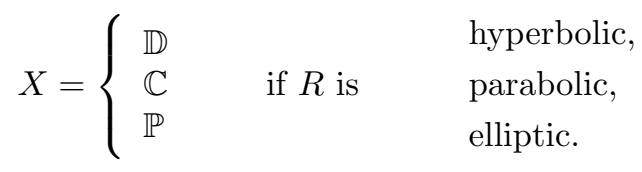

Note that the pullback $\mu=\pi^{*} \lambda$ of the conformal metric $\lambda(w)|d w|$ under $\pi: X \rightarrow R$ to the universal covering surface $X$ is a real analytic function on $\pi^{-1}(f(I))$. Fix $\eta_{0} \in X$ with $\pi\left(\eta_{0}\right)=f(0)$. Then there exists a unique continuous lift

$$
g: K_{\delta}^{+}(0) \cup(-\delta, \delta) \rightarrow X
$$

with $\pi \circ g=f$ and $g(0)=\eta_{0}$. Moreover, $g: K_{\delta}^{+}(0) \rightarrow X$ is analytic. Also,

$$
\lim _{z \rightarrow \xi} \frac{\mu(g(z))\left|g^{\prime}(z)\right|}{\left|h^{\prime}(z)\right|}=\lim _{z \rightarrow \xi} \frac{\lambda(f(z))\left|f^{\prime}(z)\right|}{\left|h^{\prime}(z)\right|}=1, \quad \xi \in(-\delta, \delta) .
$$

If $X=\mathbb{D}$ or $X=\mathbb{C}$, then we can apply Lemma 3.4 directly and conclude $g$ has an analytic continuation $G$ to a disk $K_{r}(0)$ with $r \in(0, \delta)$. Also $G\left(K_{r}(0)\right) \subseteq X$ by choosing $r>0$ sufficiently small. Thus $F:=\pi \circ G$ is an analytic continuation of $f$ to $\mathbb{H} \cup K_{r}(0)$ with $F\left(\mathbb{H} \cup K_{r}(0)\right) \subset R$.

If $X=\mathbb{P}$, then $g: K_{\delta}^{+}(0) \rightarrow \mathbb{P}$ is a meromorphic function, which is continuous from $\left(K_{\delta}^{+}(0) \cup(-\delta, \delta),|\cdot|\right)$ to $(\mathbb{P}, \mu)$. Shrinking $\delta>0$, if necessary, we may assume $g$ or $1 / g$ is holomorphic in $K_{\delta}^{+}(0)$ and continuous from $\left(K_{\delta}^{+}(0) \cup(-\delta, \delta),|\cdot|\right)$ to $(\mathbb{C}, \mu)$. In the first case we deduce as above that $g$ and therefore also $f$ has an analytic continuation $F: \mathbb{H} \cup K_{r}(0) \rightarrow R$. In the second case, we consider the 
holomorphic function $1 / g(z)$ and the pullback $\tilde{\mu}=T^{*} \mu$ of the metric $\mu(w)|d w|$ under the inversion $T(w)=1 / w$. Then from the transition relation

$$
\tilde{\mu}(1 / g(z))\left|(1 / g)^{\prime}(z)\right|=\mu(g(z))\left|g^{\prime}(z)\right|
$$

we can conclude as before that $1 / g$ has an analytic continuation to a disk $K_{r}(0)$. Thus $g$ has a meromorphic continuation to $K_{r}(0)$ and $f$ has an analytic continuation also in this case.

Proof of Theorem 3.1. We prove (a) and (b) simultaneously. Fix a point $\xi_{0} \in I$ and a number $\rho>0$ such that $I^{\prime}:=K_{\rho}\left(\xi_{0}\right) \cap \partial \mathbb{D} \subseteq I$, the function $h(z)$ is holomorphic in $K_{\rho}\left(\xi_{0}\right)$,

$$
\frac{\lambda(f(z))\left|f^{\prime}(z)\right|}{\left|h^{\prime}(z)\right|} \leq 2, \quad z \in \mathbb{D} \cap K_{\rho}\left(\xi_{0}\right)
$$

and

$$
M:=\sup _{z \in K_{\rho}\left(\xi_{0}\right)}\left|h^{\prime}(z)\right|<\infty .
$$

We are now in a position to apply Lemma 3.2 for $\Omega=K_{\rho}\left(\xi_{0}\right) \cap \mathbb{D}$. Thus $f$ extends to a continuous map from $\mathbb{D} \cup I^{\prime}$ to $R$.

Next consider the conformal map

$$
z=T(u)=-\xi_{0} \frac{u-i}{u+i}
$$

from $\mathbb{H}$ onto $\mathbb{D}$ with $T(0)=\xi_{0}$. Clearly, $T((-\delta, \delta)) \subset I^{\prime}$ for some constant $\delta>0$, so $\hat{f}:=f \circ T: \mathbb{H} \cup(-\delta, \delta) \rightarrow R$ is a continuous map, which is analytic in $\mathbb{H}$. Note $h \circ T$ is analytic in a neighborhood of $(-\delta, \delta)$ and

$$
\lim _{\substack{u \rightarrow x \\ u \in \mathbb{H}}} \frac{\lambda(\hat{f}(u))\left|\hat{f}^{\prime}(u)\right|}{\left|(h \circ T)^{\prime}(u)\right|}=\lim _{\substack{z \rightarrow T(x) \\ z \in \mathbb{D}}} \frac{\lambda(f(z))\left|f^{\prime}(z)\right|}{\left|h^{\prime}(z)\right|}=1, \quad x \in(-\delta, \delta) .
$$

By Lemma 3.5, $\hat{f}$ has an analytic continuation $\hat{F}: \mathbb{H} \cup K_{\varepsilon}(0) \rightarrow R$ to a disk $K_{\varepsilon}(0)$, $\varepsilon \in(0, \delta)$, so $f$ has an analytic continuation $F: \mathbb{D} \cup K_{r}\left(\xi_{0}\right) \rightarrow R$ to the disk $K_{r}\left(\xi_{0}\right)=T\left(K_{\varepsilon}(0)\right)$, where $r=r\left(\xi_{0}\right)>0$ is a constant depending on $\xi_{0}$. Note $K_{r}\left(\xi_{0}\right) \cap \partial \mathbb{D} \subseteq I$. It follows that $f$ has an analytic continuation to the simply connected domain

$$
\mathbb{D} \cup \bigcup_{\xi_{0} \in I} K_{r\left(\xi_{0}\right)}\left(\xi_{0}\right)
$$

3.2. Proof of the 'only if' part of Theorem 1.1. The 'only-if' part of Theorem 1.1 is contained in the following more general result.

Theorem 3.6. Let $I$ be an open subarc of the unit circle, which is contained in a simply connected domain $\Omega \supset \mathbb{D}$, and let $R$ be a Riemann surface equipped with a conformal metric $\lambda(w)|d w|$. Also, let $f: \Omega \rightarrow R$ be a non-constant analytic map, and let $\lambda(w)|d w|$ be real analytic in a neighborhood of $f(I)$. Then there exists a holomorphic function $h: I \rightarrow \mathbb{C}$ such that

$$
\frac{\lambda(f(z))\left|f^{\prime}(z)\right|}{\left|h^{\prime}(z)\right|}=1, \quad z \in I
$$


If the conformal metric $\lambda(w)|d w|$ has constant Gaussian curvature $k$, then a function $h(z)$ satisfying (3.12) can be found more or less explicitly. It therefore seems to be advisable to prove Theorem 3.6 first in this special case. Recall that the Gaussian curvature $\kappa_{\lambda}$ of the conformal metric $\lambda(w)|d w|$ is given in local coordinates by

$$
-\frac{\Delta \log \lambda(z)}{\lambda(z)^{2}}
$$

where $\Delta$ is the Laplace-Beltrami operator

$$
\Delta=\frac{\partial^{2}}{\partial x^{2}}+\frac{\partial^{2}}{\partial y^{2}}, \quad z=x+i y
$$

Proof of Theorem 3.6. (For metrics of constant Gaussian curvature $k$.)

We may assume $k \in\{-4,0,4\}$. Let $X=\mathbb{D}, \mathbb{C}$ or $\mathbb{P}$ be the universal covering surface of $R$ and let $\pi: X \rightarrow R$ be an analytic universal cover projection. Then $f$ lifts to a non-constant analytic map $g: \Omega \rightarrow X$ with $\pi \circ g=f$. Since $\mu=\pi^{*} \lambda$ is a conformal metric of constant Gaussian curvature $k \in\{-4,0,+4\}$ on the simply connected set $X$, we can apply a classical result due to Liouville (see [16, 15, 21]) which guarantees that there exists a locally univalent function $\varphi: X \rightarrow Y$ such that

where

$$
\mu(\xi)=\frac{\left|\varphi^{\prime}(\xi)\right|}{1+\frac{k}{4}|\varphi(\xi)|^{2}}, \quad \xi \in X
$$

$$
Y=\left\{\begin{array}{ll}
\mathbb{D} \\
\mathbb{C} \\
\mathbb{P}
\end{array} \quad \text { if } \quad \begin{array}{l}
k=-4, \\
k=0, \\
k=+4 .
\end{array}\right.
$$

Next consider the holomorphic (or meromorphic) function $\Psi:=\varphi \circ g: \Omega \rightarrow Y$. Since $\Psi: \Omega \rightarrow Y$ is a non-constant analytic map, there exists a simply connected domain $U$ with $I \subset U \subset \Omega$, which is symmetrid 3 about $I$, and such that $\Psi$ is a locally univalent holomorphic function in $U \backslash I$. Thus we can define a holomorphic function $h: U \rightarrow \mathbb{C}$ by means of the formula

$$
h^{\prime}(z):=\frac{\sqrt{\Psi^{\prime}(z) \overline{\Psi^{\prime}(1 / \bar{z})}}}{1+\frac{k}{4} \Psi(z) \overline{\Psi(1 / \bar{z})}}, \quad z \in U
$$

Now it easily follows that

$$
\frac{\left|\Psi^{\prime}(z)\right|}{1+\frac{k}{4}|\Psi(z)|^{2}} \frac{1}{\left|h^{\prime}(z)\right|}=1, \quad z \in I
$$

or, in other 'words',

$$
\frac{\lambda(f(z))\left|f^{\prime}(z)\right|}{\left|h^{\prime}(z)\right|}=\frac{\mu(g(z))\left|g^{\prime}(z)\right|}{\left|h^{\prime}(z)\right|}=\frac{\left|\Psi^{\prime}(z)\right|}{1+\frac{k}{4}|\Psi(z)|^{2}} \frac{1}{\left|h^{\prime}(z)\right|}=1, \quad z \in I .
$$

Now, we turn to the proof of Theorem 3.6 for general conformal metrics $\lambda(w)|d w|$.

\footnotetext{
${ }^{3} z \in U$ implies $1 / \bar{z} \in U$.
} 
Proof of Theorem 3.6. Let $X=\mathbb{D}, \mathbb{C}$ or $\mathbb{P}$ be the universal covering surface of $R$ and let $\pi: X \rightarrow R$ be an analytic universal covering map. The non-constant analytic map $f$ lifts to a non-constant meromorphic function $g: \Omega \rightarrow X$ with $\pi \circ g=f$. Moreover, the pullback $\mu(v)|d v|=\left(\pi^{*} \lambda\right)(v)|d v|$ of the real analytic metric $\lambda(w)|d w|$ to $X$ under $w=\pi(v)$ is a conformal metric on the covering surface $X$, which is real analytic in a neighborhood of $g(I)$. If $X=\mathbb{D}$ or $X=\mathbb{C}$, then we identify the metric $\mu(v)|d v|$ with the function $\mu_{0}: X \rightarrow \mathbb{R}$ associated with the local coordinate $\varphi_{0}(v)=v$. If $X=\mathbb{P}$, the Riemann sphere, then $\varphi_{\infty}(v)=1 / v$ is a local parameter from $\mathbb{P} \backslash\{0\}$ onto $\mathbb{C}$, and $\varphi_{0}(v)=v$ is a local coordinate from $\mathbb{C}$ onto $\mathbb{C}$. Also, we may assume $\mu(v)|d v|$ is given by the family $\left(\mu_{\infty}, \mu_{0}\right)$ of positive functions $\mu_{\infty}: \mathbb{C} \rightarrow \mathbb{R}$ and $\mu_{0}: \mathbb{C} \rightarrow \mathbb{R}$, which are related by the transition rule

$$
\mu_{0}(v)=\mu_{\infty}(1 / v) \frac{1}{|v|^{2}}, \quad v \in \mathbb{C} \backslash\{0\}
$$

Since $g: \Omega \rightarrow X$ is a non-constant analytic map, there exists a simply connected domain $U$ with $I \subset U \subset \Omega$, which is symmetric about $I$, and such that $g$ is a locally univalent holomorphic function in $U \backslash I$. In particular, $g$ has its poles in $U$ only on $I$. Consequently, the function $g^{\prime}(z) \overline{g^{\prime}(1 / \bar{z})}$ is a meromorphic function in $U$, which has zeros and poles only on the arc $I$. These zeros and poles are therefore of even order. Thus we can define a meromorphic square root

$$
\sqrt{g^{\prime}(z) \overline{g^{\prime}(1 / \bar{z})}}
$$

of the meromorphic function $g^{\prime}(z) \overline{g^{\prime}(1 / \bar{z})}$ in $U$.

In the next step we are going to construct for each $\xi \in I$ a holomorphic function $h_{\xi}$ in a disk $K_{r(\xi)}(\xi) \subset U$ such that

$$
\frac{\lambda(f(z))\left|f^{\prime}(z)\right|}{\left|h_{\xi}^{\prime}(z)\right|}=1, \quad z \in K_{r(\xi)}(\xi) \cap I .
$$

Shrinking the disks $K_{r(\xi)}(\xi)$, if necessary, we may assume

$$
V:=\bigcup_{\xi \in I} K_{r(\xi)}(\xi)
$$

is a simply connected domain and $K_{r\left(\xi_{0}\right)}\left(\xi_{0}\right) \cap K_{r\left(\xi_{1}\right)}\left(\xi_{1}\right) \neq \emptyset$ always implies $K_{r\left(\xi_{0}\right)}\left(\xi_{0}\right) \cap K_{r\left(\xi_{1}\right)}\left(\xi_{1}\right) \cap I \neq \emptyset$. We then show

$$
h_{\xi_{0}}^{\prime} \equiv h_{\xi_{1}}^{\prime} \quad \text { in } K_{r\left(\xi_{0}\right)}\left(\xi_{0}\right) \cap K_{r\left(\xi_{1}\right)}\left(\xi_{1}\right)
$$

for all $\xi_{0}, \xi_{1} \in I$. Thus the locally defined holomorphic functions $h_{\xi}, \xi \in I$, can be patched together along $I$ to a holomorphic function $h: V \rightarrow \mathbb{C}$, which satisfies the boundary condition (3.12).

We begin with the local construction of the holomorphic function $h(z)$. Fix a point $\xi \in I$ and let $v=g(\xi) \in X$. We have to distinguish the cases $v \neq \infty$ and $v=\infty$. In the first case $v \neq \infty$, let $\zeta=\operatorname{Re} v$ and $\eta=\operatorname{Im} v$. Since $\mu_{0}$ is real analytic in a neighborhood of $(\zeta, \eta) \in \mathbb{R}^{2}$ it has a holomorphic extension $\Phi_{\xi}$ to the set $K_{\rho}(\zeta) \times K_{\rho}(\eta) \subset \mathbb{C}^{2}$ for some $\rho=\rho(\xi)>0$ such that

$$
\Phi_{\xi}(x, y)=\mu_{0}(x+i y)
$$

for $x \in K_{\rho}(\zeta) \cap \mathbb{R}$ and $y \in K_{\rho}(\eta) \cap \mathbb{R}$. We also note

$$
\frac{1}{2}(g(z)+\overline{g(1 / \bar{z})}) \in K_{\rho}(\zeta), \quad \frac{1}{2 i}(g(z)-\overline{g(1 / \bar{z})}) \in K_{\rho}(\eta),
$$


for all $z \in K_{r}(\xi) \subset U$ for some $r=r(\xi)>0$. In particular, $g$ is a holomorphic function on $K_{r}(\xi)$. Now we can define a holomorphic function $h_{\xi}^{\prime}: K_{r}(\xi) \rightarrow \mathbb{C}$ by

$$
h_{\xi}^{\prime}(z):=\Phi_{\xi}\left(\frac{1}{2}(g(z)+\overline{g(1 / \bar{z})}), \frac{1}{2 i}(g(z)-\overline{g(1 / \bar{z})})\right) \sqrt{g^{\prime}(z) \overline{g^{\prime}(1 / \bar{z})}} .
$$

Clearly,

$$
\frac{\lambda(f(z))\left|f^{\prime}(z)\right|}{\left|h_{\xi}^{\prime}(z)\right|}=\frac{\mu_{0}(g(z))\left|g^{\prime}(z)\right|}{\left|h_{\xi}^{\prime}(z)\right|}=\frac{\mu_{0}(g(z))\left|g^{\prime}(z)\right|}{\left|\Phi_{\xi}(\operatorname{Re} g(z), \operatorname{Im} g(z))\right|\left|\sqrt{\left|g^{\prime}(z)\right|^{2}}\right|}=1
$$

for $z \in K_{r}(\xi) \cap I$.

If $v=\infty$, then we define $h_{\xi}$ in a similar way but using $\mu_{\infty}$ instead of $\mu_{0}$. Here are the details. Since $\mu_{\infty}$ is real analytic in a neighborhood of $(0,0) \in \mathbb{R}^{2}$, it has a holomorphic extension $\Phi_{\infty}$ to the set $K_{\rho}(0) \times K_{\rho}(0) \subset \mathbb{C}^{2}$ for some $\rho>0$ such that

$$
\Phi_{\infty}(x, y)=\mu_{\infty}(x+i y)
$$

for $x, y \in K_{\rho}(0) \cap \mathbb{R}$. Then

$$
\frac{1}{2}\left(\frac{1}{g(z)}+\frac{1}{\overline{g(1 / \bar{z})}}\right) \in K_{\rho}(0), \quad \frac{1}{2 i}\left(\frac{1}{g(z)}-\frac{1}{\overline{g(1 / \bar{z})}}\right) \in K_{\rho}(0)
$$

for all $z \in K_{r}(\xi) \subset U$ for some $r=r(\xi)>0$. Also, we may assume by shrinking $r>0$, if necessary, that $g$ has a pole in $K_{r}(\xi)$ only at the point $\xi$. Further note

$$
\left(\frac{1}{g}\right)^{\prime}=-\frac{g^{\prime}}{g^{2}} \neq 0 \quad \text { in } K_{r}(\xi) \backslash I
$$

Now we can define a holomorphic function $h_{\xi}^{\prime}: K_{r}(\xi) \rightarrow \mathbb{C}$ by

$$
h_{\xi}^{\prime}(z):=\Phi_{\infty}\left(\frac{1}{2}\left(\frac{1}{g(z)}+\frac{1}{\overline{g(1 / \bar{z})}}\right), \frac{1}{2 i}\left(\frac{1}{g(z)}-\frac{1}{\overline{g(1 / \bar{z})}}\right)\right) \frac{\sqrt{g^{\prime}(z) \overline{g^{\prime}(1 / \bar{z})}}}{g(z) \overline{g(1 / \bar{z})}}
$$

For $z \in K_{r}(\xi) \cap I, z \neq \xi$, this yields

$$
\begin{aligned}
\left|h_{\xi}^{\prime}(z)\right| & =\left|\Phi_{\infty}\left(\operatorname{Re} \frac{1}{g(z)}, \operatorname{Im} \frac{1}{g(z)}\right)\right| \frac{\left|g^{\prime}(z)\right|}{|g(z)|^{2}}=\mu_{\infty}(1 / g(z)) \frac{1}{|g(z)|^{2}}\left|g^{\prime}(z)\right| \\
& =\mu_{0}(g(z))\left|g^{\prime}(z)\right|=\lambda(f(z))\left|f^{\prime}(z)\right| .
\end{aligned}
$$

So

$$
\frac{\lambda(f(z))\left|f^{\prime}(z)\right|}{\left|h_{\xi}^{\prime}(z)\right|}=1
$$

for all $z \in K_{r}(\xi) \cap I$.

We have thus constructed for each $\xi \in I$ a holomorphic function $h_{\xi}^{\prime}: K_{r(\xi)}(\xi) \rightarrow$ $\mathbb{C}$, which satisfies the boundary condition (3.14). Shrinking the disks $K_{r(\xi)}(\xi)$, if necessary, we may assume

$$
V:=\bigcup_{\xi \in I} K_{r(\xi)}(\xi)
$$

is a simply connected domain and $K_{r\left(\xi_{0}\right)}\left(\xi_{0}\right) \cap K_{r\left(\xi_{1}\right)}\left(\xi_{1}\right) \neq \emptyset$ for $\xi_{0}, \xi_{1} \in I$ implies $K_{r\left(\xi_{0}\right)}\left(\xi_{0}\right) \cap K_{r\left(\xi_{1}\right)}\left(\xi_{1}\right) \cap I \neq \emptyset$. 
We now claim $h_{\xi_{0}}^{\prime} \equiv h_{\xi_{1}}^{\prime}$ in $W:=K_{r\left(\xi_{0}\right)}\left(\xi_{0}\right) \cap K_{r\left(\xi_{1}\right)}\left(\xi_{1}\right)$ for all $\xi_{0}, \xi_{1} \in I$ such that $W \neq \emptyset$. The following four cases can occur:

$$
\begin{aligned}
\text { (I) } \quad g\left(\xi_{0}\right) \neq \infty, g\left(\xi_{1}\right) \neq \infty, \quad(\mathrm{II}) \quad g\left(\xi_{0}\right)=\infty, g\left(\xi_{1}\right) \neq \infty \\
\text { (III) } g\left(\xi_{0}\right) \neq \infty, g\left(\xi_{1}\right)=\infty, \quad(\mathrm{IV}) \quad g\left(\xi_{0}\right)=\infty, g\left(\xi_{1}\right)=\infty
\end{aligned}
$$

We only consider (I) and (II). The cases (III) and (IV) can be handled analogously.

(I) Let $\zeta_{0}=\operatorname{Re} g\left(\xi_{0}\right), \eta_{0}=\operatorname{Im} g\left(\xi_{0}\right)$, and $\zeta_{1}=\operatorname{Re} g\left(\xi_{1}\right), \eta_{1}=\operatorname{Im} g\left(\xi_{1}\right)$. As we have seen above $\mu_{0}$ has a holomorphic extension $\Phi_{\xi_{0}}$ to the set $U_{0}:=K_{\rho\left(\xi_{0}\right)}\left(\zeta_{0}\right) \times$ $K_{\rho\left(\xi_{0}\right)}\left(\eta_{0}\right) \subset \mathbb{C}^{2}$ and a holomorphic extension $\Phi_{\xi_{1}}$ to the set $U_{1}:=K_{\rho\left(\xi_{1}\right)}\left(\zeta_{1}\right) \times$ $K_{\rho\left(\xi_{1}\right)}\left(\eta_{1}\right) \subset \mathbb{C}^{2}$. We claim

(i) $U_{0} \cap U_{1} \neq \emptyset$, and

(ii) $\Phi_{\xi_{0}} \equiv \Phi_{\xi_{1}}$ in $U_{0} \cap U_{1}$.

To prove (i) observe that for $g(z)=\zeta+i \eta$ with $z \in W \cap I$, which is a non-empty set by construction, we have

$$
\begin{aligned}
\zeta & =\frac{1}{2}(g(z)+\overline{g(1 / \bar{z})}) \in K_{\rho\left(\xi_{0}\right)}\left(\zeta_{0}\right) \cap K_{\rho\left(\xi_{1}\right)}\left(\zeta_{1}\right) \cap \mathbb{R} \\
\eta & =\frac{1}{2 i}(g(z)-\overline{g(1 / \bar{z})}) \in K_{\rho\left(\xi_{0}\right)}\left(\eta_{0}\right) \cap K_{\rho\left(\xi_{1}\right)}\left(\eta_{1}\right) \cap \mathbb{R}
\end{aligned}
$$

by (3.15), so $(\zeta, \eta) \in U_{0} \cap U_{1}$.

To prove (ii) note that

$$
\Phi_{\xi_{0}}(x, y)=\mu_{0}(x+i y)=\Phi_{\xi_{1}}(x, y)
$$

for all $x \in K_{\rho\left(\xi_{0}\right)}\left(\zeta_{0}\right) \cap K_{\rho\left(\xi_{1}\right)}\left(\zeta_{1}\right) \cap \mathbb{R}$ and all $y \in K_{\rho\left(\xi_{0}\right)}\left(\eta_{0}\right) \cap K_{\rho\left(\xi_{1}\right)}\left(\eta_{1}\right) \cap \mathbb{R}$, which implies (ii). Consequently, since

$$
\left(\frac{1}{2}(g(z)+\overline{g(1 / \bar{z})}), \frac{1}{2 i}(g(z)-\overline{g(1 / \bar{z})})\right) \in U_{0} \cap U_{1},
$$

for $z \in W$ in view of (3.15), we obtain

$$
\begin{aligned}
h_{\xi_{0}}^{\prime}(z) & =\Phi_{\xi_{0}}\left(\frac{1}{2}(g(z)+\overline{g(1 / \bar{z})}), \frac{1}{2 i}(g(z)-\overline{g(1 / \bar{z})})\right) \sqrt{g^{\prime}(z) \overline{g^{\prime}(1 / \bar{z})}} \\
& =\Phi_{\xi_{1}}\left(\frac{1}{2}(g(z)+\overline{g(1 / \bar{z})}), \frac{1}{2 i}(g(z)-\overline{g(1 / \bar{z})})\right) \sqrt{g^{\prime}(z) \overline{g^{\prime}(1 / \bar{z})}} \\
& =h_{\xi_{1}}^{\prime}(z)
\end{aligned}
$$

for every $z \in W$.

We finally consider the case (II) and let $\zeta_{1}=\operatorname{Re} g\left(\xi_{1}\right)$ and $\eta_{1}=\operatorname{Im} g\left(\zeta_{1}\right)$. Then $\mu_{0}$ has a holomorphic extension $\Phi_{\xi_{1}}$ to $U_{1}:=K_{\rho\left(\xi_{1}\right)}\left(\zeta_{1}\right) \times K_{\rho\left(\xi_{1}\right)}\left(\eta_{1}\right) \subset \mathbb{C}^{2}$, and $\mu_{\infty}$ has a holomorphic extension $\Phi_{\infty}$ to $U_{\infty}:=K_{\rho\left(\xi_{0}\right)}(0) \times K_{\rho\left(\xi_{0}\right)}(0) \subset \mathbb{C}^{2}$. Also note that $\mu_{0}$ and $\mu_{\infty}$ are related by (3.13). Let

We claim

$$
U_{0}:=\left\{(\zeta, \eta) \in \mathbb{C}^{2}:\left(\frac{\zeta}{\zeta^{2}+\eta^{2}},-\frac{\eta}{\zeta^{2}+\eta^{2}}\right) \in U_{\infty}\right\}
$$

(i) $U_{0} \cap U_{1} \neq \emptyset$ and

(ii)

$$
\Phi_{\infty}\left(\frac{\zeta}{\zeta^{2}+\eta^{2}},-\frac{\eta}{\zeta^{2}+\eta^{2}}\right) \frac{1}{\zeta^{2}+\eta^{2}}=\Phi_{\xi_{1}}(\zeta, \eta) \text { for all }(\zeta, \eta) \in U_{0} \cap U_{1}
$$


To prove (i) let $z \in W \cap I$ and $g(z)=\zeta+i \eta$. Then, in view of (3.15), it is easy to see $(\zeta, \eta) \in U_{1}$, and also

$$
\begin{aligned}
\frac{\zeta}{\zeta^{2}+\eta^{2}} & =\frac{1}{2}\left(\frac{1}{g(z)}+\frac{1}{\overline{g(1 / \bar{z})}}\right) \in K_{\rho\left(\xi_{0}\right)}(0), \\
-\frac{\eta}{\zeta^{2}+\eta^{2}} & =\frac{1}{2 i}\left(\frac{1}{g(z)}-\frac{1}{\overline{g(1 / \bar{z})}}\right) \in K_{\rho\left(\xi_{0}\right)}(0),
\end{aligned}
$$

by (3.16), so $(\zeta, \eta) \in U_{0}$.

To establish (ii) let $(x, y) \in U_{0} \cap U_{1}$ with $x, y \in \mathbb{R}$. Then

$$
\left(\frac{x}{x^{2}+y^{2}},-\frac{y}{x^{2}+y^{2}}\right) \in U_{\infty}
$$

so

$$
\begin{aligned}
\Phi_{\infty} & \left(\frac{x}{x^{2}+y^{2}},-\frac{y}{x^{2}+y^{2}}\right) \frac{1}{x^{2}+y^{2}} \\
& =\mu_{\infty}\left(\frac{x-i y}{x^{2}+y^{2}}\right) \frac{1}{x^{2}+y^{2}} \\
& =\mu_{\infty}\left(\frac{1}{x+i y}\right) \frac{1}{|x+i y|^{2}} \\
& =\mu_{0}(x+i y)=\Phi_{\xi_{1}}(x, y),
\end{aligned}
$$

which proves (ii).

Now, if $z \in W$, then

$$
\left(\frac{1}{2}(g(z)+\overline{g(1 / \bar{z})}), \frac{1}{2 i}(g(z)-\bar{g}(1 / \bar{z}))\right) \in U_{0} \cap U_{1},
$$

since

$$
\begin{gathered}
\frac{1}{2}(g(z)+\overline{g(1 / \bar{z})}) \in K_{\rho\left(\xi_{1}\right)}\left(\zeta_{1}\right), \\
\frac{1}{2 i}(g(z)-\overline{g(1 / \bar{z})}) \in K_{\rho\left(\xi_{1}\right)}\left(\eta_{1}\right), \\
\frac{1}{2}(g(z)+\overline{g(1 / \bar{z})}) \\
=\frac{\frac{1}{2}(g(z)+\overline{g(1 / \bar{z})})}{g(z) \overline{g(1 / \bar{z})}}=\frac{1}{2}\left(\frac{1}{g(z)}+\frac{1}{\overline{g(1 / \bar{z})}}\right) \in K_{\rho\left(\xi_{0}\right)}(0)
\end{gathered}
$$

and

$$
\begin{gathered}
-\frac{\frac{1}{2 i}(g(z)-\overline{g(1 / \bar{z})})}{\left(\frac{1}{2}(g(z)+\overline{g(1 / \bar{z})})\right)^{2}+\left(\frac{1}{2 i}(g(z)-\overline{g(1 / \bar{z})})\right)^{2}} \\
\quad=-\frac{\frac{1}{2 i}(g(z)-\overline{g(1 / \bar{z})})}{g(z) \overline{g(1 / \bar{z})}}=\frac{1}{2 i}\left(\frac{1}{g(z)}-\frac{1}{\overline{g(1 / \bar{z})}}\right) \in K_{\rho\left(\xi_{0}\right)}(0) .
\end{gathered}
$$


Thus by (ii)

$$
\begin{aligned}
h_{\xi_{1}}^{\prime}(z) & =\Phi_{\xi_{1}}\left(\frac{1}{2}(g(z)+\overline{g(1 / \bar{z})}), \frac{1}{2 i}(g(z)-\overline{g(1 / \bar{z})})\right) \sqrt{g^{\prime}(z) \overline{g^{\prime}(1 / \bar{z})}} \\
& =\Phi_{\infty}\left(\frac{1}{2}\left(\frac{1}{g(z)}+\frac{1}{\overline{g(1 / \bar{z})}}\right), \frac{1}{2 i}\left(\frac{1}{g(z)}-\frac{1}{\overline{g(1 / \bar{z})}}\right)\right) \frac{\sqrt{g^{\prime}(z) \overline{g^{\prime}(1 / \bar{z})}}}{g(z) \overline{g(1 / \bar{z})}} \\
& =h_{\xi_{0}}^{\prime}(z)
\end{aligned}
$$

for all $z \in W$.

We have proved that $h_{\xi_{0}}^{\prime} \equiv h_{\xi_{1}}^{\prime}$ in $K_{r\left(\xi_{0}\right)}\left(\xi_{0}\right) \cap K_{r\left(\xi_{1}\right)}\left(\xi_{1}\right)$ for all $\xi_{0}, \xi_{1} \in I$. We can thus define a holomorphic function $h^{\prime}$ on the simply connected domain

$$
V=\bigcup_{\xi \in I} K_{r(\xi)}(\xi)
$$

via

$$
h^{\prime}(z)=h_{\xi}^{\prime}(z), \quad z \in K_{r(\xi)}(\xi),
$$

which by construction satisfies the condition (3.12).

3.3. Proof of Theorem 1.10, We already know that $f$ has a continuous extension to $\overline{\mathbb{D}}$, which we also denote by $f$. Thus $\Phi(f(z)) \geq \varepsilon$ on $|z|=1$ for some constant $\varepsilon>0$. Also, the function

$$
\lambda(w):=\frac{1}{\Phi(w)}
$$

is positive and continuous on $\mathbb{C}$, and real analytic in a neighborhood of $f(I)$. Moreover,

$$
\lim _{z \rightarrow \xi} \lambda(f(z))\left|f^{\prime}(z)\right|=\lim _{z \rightarrow \xi} \frac{\left|f^{\prime}(z)\right|}{\Phi(f(z))}=1+\lim _{z \rightarrow \xi} \frac{\left|f^{\prime}(z)\right|-\Phi(f(z))}{\Phi(f(z))}=1, \quad \xi \in I,
$$

in view of (1.7). Thus we can apply Theorem 3.1(b) with $h(z) \equiv z$ and conclude that $f$ has an analytic continuation across $I$.

3.4. Proof of Proposition 1.11. By assumption, $\Psi$ is a holomorphic function in the disk $K_{\rho}(0)$ for some $\rho>1$ and $\Psi:[0,1] \rightarrow(0,1)$. Therefore the function

$$
\lambda(w):=\frac{1}{\Psi\left(|w|^{2}\right)}
$$

is well defined and real analytic in the disk $|w| \leq r$ for some $r \in(1, \rho)$ and $\lambda(w) \geq$ $\delta>0$ there. We can extend $\lambda(w)$ to a $C^{\infty}$ function on all of $\mathbb{C}$, which is still bounded from below by a positive constant; also call this extension $\lambda(w)$. Then $\lambda(w)|d w|$ is a complete conformal Riemannian metric on the complex plane, which is real analytic in a neighborhood of $\overline{\mathbb{D}}$. Moreover,

$$
\lim _{z \rightarrow \xi} \lambda(F(z))\left|F^{\prime}(z)\right|=1, \quad \xi \in \partial \mathbb{D},
$$

by (1.8). Thus Theorem 3.1 is applicable and shows that $F$ has a holomorphic extension to a neighborhood of $\overline{\mathbb{D}}$. 
3.5. Proof of Theorem 1.12, The proof of Theorem 1.12 is similar to the proof of Theorem 1.1, so we only give a quick outline. Details are left to the reader.

First we note that we may assume $f(z)$ is a holomorphic function in the upper half plane, $h(z)$ is a holomorphic function on $I=(-\delta, \delta)$ for some $\delta>0$, and

$$
\lim _{z \rightarrow \xi} \frac{\lambda(f(z))\left|f^{\prime}(z)\right|}{\mu(h(z))\left|h^{\prime}(z)\right|}=1
$$

for every $\xi \in(-\delta, \delta)$. Using the Hopf-Rinow Theorem 2.1 we see that (3.19) implies $f$ has a continuous extension to the interval $(-r, r)$ for some $r>0$. Then, again using (3.19), we deduce that $f^{\prime}(z)$ and $h^{\prime}(z) / f^{\prime}(z)$ are bounded holomorphic functions in $K_{r}^{+}(0)$. Thus Fatou's Theorem 2.3 is applicable and shows $f^{\prime}(z)$ and $h^{\prime}(z) / f^{\prime}(z)$ have vertical limits $f^{\prime}(x)$ and $h^{\prime}(x) / f^{\prime}(x)$ for almost every $x \in(-r, r)$. Moreover, $f^{\prime}(x)$ and $h^{\prime}(x) / f^{\prime}(x)$ belong to $L^{\infty}(-r, r)$. The boundary condition (3.19) now takes the form

$$
\frac{d}{d x} \overline{f(x)}=\frac{h^{\prime}(x)}{f^{\prime}(x)} \overline{h^{\prime}(x)} \frac{\mu(h(x))^{2}}{\lambda(f(x))^{2}}
$$

for almost every $x \in(-r, r)$. As in the proof of Lemma 3.4 we can write this equation as

$$
\frac{d}{d x} \overline{f(x)}=\frac{h^{\prime}(x)}{f^{\prime}(x)} \overline{h^{\prime}(x)} \Phi(\operatorname{Re} h(x), \operatorname{Im} h(x), \operatorname{Re} f(x), \operatorname{Im} f(x)) \quad \text { for a.e. } x \in(-r, r),
$$

where $\Phi: U \rightarrow \mathbb{C}$ is a holomorphic function in a neighborhood $U \subset \mathbb{C}^{4}$ of the point $(\operatorname{Re} h(0), \operatorname{Im} h(0), \operatorname{Re} f(0), \operatorname{Im} f(0)) \in \mathbb{C}^{4}$.

If $f$ has an analytic continuation $F$ to $K_{r}^{-}(0)$, then $g(z)=\overline{F(\bar{z})}$ is a solution of the initial value problem

$$
g^{\prime}(z)=\frac{h^{\prime}(z)}{f^{\prime}(z)} \overline{h^{\prime}(\bar{z})} \Phi\left(\frac{h(z)+\overline{h(\bar{z})}}{2}, \frac{h(z)-\overline{h(\bar{z})}}{2 i}, \frac{f(z)+g(z)}{2}, \frac{f(z)-g(z)}{2 i}\right),
$$

$$
g(0)=\overline{f(0)} \text {. }
$$

Again this observation is reversible. Using Lemma 2.4, we find a solution $g$ : $\overline{K_{\varepsilon}^{+}(0)} \rightarrow \mathbb{C}$ of the initial value problem (3.20)-3.21). It can then be shown that $g(z)$ is a holomorphic function in $K_{\varepsilon}^{+}(0)$, which agrees with $\overline{f(z)}$ on the real axis. In other words, $F(z):=\overline{g(\bar{z})}, z \in K_{\varepsilon}^{-}(0)$, is the analytic continuation of $f(z)$ to the lower half of the disk $K_{\varepsilon}(0)$.

\section{Appendix}

4.1. Some generalizations of Theorem 1.1. In this section, we state and partly prove some generalizations of Theorem 1.1 and Theorem 1.12 .

It is pretty obvious that the unit disk $\mathbb{D}$ plays no special role in Theorem 1.1 - we can replace it by any domain $D$ in the complex plane with a 'free analytic boundary arc'. Roughly speaking, a free analytic boundary arc $I$ of a domain $D \subset \mathbb{C}$ is an analytic Jordan arc which divides each sufficiently small disk centered on $I$ into two simply connected domains, one and only one of which belongs to $D$. We recall the following more formal definition. 
Definition 4.1. An (open) connected proper subset $I$ of the boundary of a domain $D$ is called an (open) free analytic boundary arc of $D$, if for every $\xi_{0} \in I$ there is a neighborhood $U$ of $\xi_{0}$ and a conformal map $\Psi$ from $\mathbb{D}$ onto $U$ such that

(a) $\Psi(0)=\xi_{0}$,

(b) $\Psi((-1,1))=I \cap U$,

(c) $\Psi(\mathbb{D} \cap \mathbb{H})=D \cap U$.

We can now state the following local extension of Theorem 1.12

Theorem 4.1. Let $D$ be a domain in the complex plane, let $I$ be a free analytic boundary arc of $D$, and let $R$ be a Riemann surface, which carries a complete real analytic conformal metric $\lambda(w)|d w|$. Also, let $h: I \rightarrow \mathbb{C}$ be a holomorphic function, and let $\mu$ be a positive and real analytic function on a neighborhood of $h(I)$. If $f: D \rightarrow R$ is an analytic mapping such that

$$
\lim _{z \rightarrow \xi} \frac{\lambda(f(z))\left|f^{\prime}(z)\right|}{\mu(h(z))\left|h^{\prime}(z)\right|}=1, \quad \xi \in I,
$$

then $f$ can be continued analytically across any compact subset $J$ of $I$ with $f(J) \subset$ $R$.

Proof. Let $\xi_{0} \in J$ and let $z=\Psi(\zeta)$ be a conformal map from $\mathbb{D}$ onto a neighborhood $U$ of $\xi_{0}$ such that $\Psi(0)=\xi_{0}, \Psi((-1,1))=I \cap U$ and $\Psi(\mathbb{D} \cap \mathbb{H})=D \cap U$. Thus $g:=f \circ \Psi$ is an analytic function in $K_{1}^{+}(0)$ and satisfies

$$
\lim _{\zeta \rightarrow x} \frac{\lambda(g(\zeta))\left|g^{\prime}(\zeta)\right|}{\mu(h(\Psi(\zeta)))\left|(h \circ \Psi)^{\prime}(\zeta)\right|}=\lim _{z \rightarrow \xi} \frac{\lambda(f(z))\left|f^{\prime}(z)\right|}{\mu(h(z))\left|h^{\prime}(z)\right|}=1, \quad x \in(-1,1) .
$$

It follows (see the proof of Theorem 1.12) that $g$ has an analytic continuation $G$ to the disk $K_{r}(0)$ for some $r>0$ such that $G\left(K_{r}(0)\right) \subseteq R$. Thus, $f$ has an analytic continuation $F=G \circ \Psi^{-1}$ to a neighborhood $V\left(\xi_{0}\right) \subseteq U$ of $\xi_{0}$ with $F\left(V\left(\xi_{0}\right)\right) \subseteq R$.

The open sets $V(\xi), \xi \in J$, form an open cover of the compact set $J$. It is thus possible to extract a finite subcover $V_{1}, \ldots, V_{n}$ with corresponding analytic extension $F_{j}: D \cup V_{j} \rightarrow R$ of $f$. Without loss of generality we may assume $V_{i} \cap V_{j} \cap D \neq \emptyset$, whenever $V_{i} \cap V_{j} \neq \emptyset$. Hence, if $V_{i} \cap V_{j} \neq \emptyset$, then $F_{i}$ and $F_{j}$ agree on $V_{i} \cap V_{j}$, since they agree on $V_{i} \cap V_{j} \cap D \neq \emptyset$. Therefore, we obtain an analytic extension $F$ of $f$ to the domain

$$
\Omega:=D \cup \bigcup_{j=1}^{n} V_{j}
$$

with $F(\Omega) \subseteq R$.

We also have a global extension of Theorem 1.1

Theorem 4.2. Let $D$ be a simply connected Jordan domain in the complex plane and let $I$ be an open free analytic boundary arc of D. Moreover, let $R$ be a Riemann surface, which carries a complete real analytic conformal metric $\lambda(w)|d w|$. Then a non-constant analytic mapping $f: D \rightarrow R$ can be continued analytically across $I$ with $f(I) \subset R$ if and only if there exists a holomorphic function $h: I \rightarrow \mathbb{C}$ such that

$$
\lim _{z \rightarrow \xi} \frac{\lambda(f(z))\left|f^{\prime}(z)\right|}{\left|h^{\prime}(z)\right|}=1, \quad \xi \in I .
$$

\footnotetext{
${ }^{4}$ Replace $V_{i}$ and $V_{j}$ by smaller open sets, if necessary.
} 
Proof. Let $z=T(\zeta)$ be a conformal map from $\mathbb{D}$ onto $D$. Then $T$ extends to a homeomorphism from $\overline{\mathbb{D}}$ onto $\bar{D}$. Let $I_{1}:=T^{-1}(I)$ be the open subarc of $\partial \mathbb{D}$ which is mapped by $T$ onto $I$. By the Schwarz reflection principle there are domains $\Omega_{1}$ and $\Omega$ with $\mathbb{D} \cup I_{1} \subset \Omega_{1}$ and $D \cup I \subset \Omega$ such that $T$ extends to a conformal map from $\Omega_{1}$ onto $\Omega$.

If $f: D \rightarrow R$ is an analytic map and (4.1) holds for every $\xi \in I$ for some holomorphic function $h: I \rightarrow \mathbb{C}$, then $h_{1}:=h \circ T$ is a holomorphic function on $I_{1}$, $f_{1}:=f \circ T$ is an analytic map from $\mathbb{D}$ into $R$, and

$$
\lim _{\zeta \rightarrow \eta} \frac{\lambda\left(f_{1}(\zeta)\right)\left|f_{1}^{\prime}(\zeta)\right|}{\left|h_{1}^{\prime}(\zeta)\right|}=\lim _{z \rightarrow \xi} \frac{\lambda(f(z))\left|f^{\prime}(z)\right|}{\left|h^{\prime}(z)\right|}=1
$$

for every $\eta=T^{-1}(\xi) \in I_{1}$. We are now in a position to apply the 'if' part of Theorem 1.1. which implies that $f_{1}$ can be continued analytically across $I_{1}$ to an analytic map $F_{1}$ from a neighborhood $U_{1} \subset \Omega_{1}$ of $I$ into $R$. Then $F:=F_{1} \circ T^{-1}$ is the analytic continuation of $f$ across $I$.

If, on the other hand, $f$ has an analytic continuation $F: U \rightarrow R$ to some neighborhood $U \subset \Omega$ of $I$, then $f_{1}$ has the analytic continuation $F_{1}:=F \circ T$ to the neighborhood $U_{1}=T^{-1}(U)$ of $I_{1}$. We can apply the 'only if' part of Theorem 1.1 and obtain a holomorphic function $h_{1}: I_{1} \rightarrow \mathbb{C}$ such that

$$
\frac{\lambda\left(F_{1}(\zeta)\right)\left|F_{1}^{\prime}(\zeta)\right|}{\left|h_{1}^{\prime}(\zeta)\right|}=1
$$

for every $\zeta \in I_{1}$. This shows

$$
\frac{\lambda(F(z))\left|F^{\prime}(z)\right|}{\left|h^{\prime}(z)\right|}=1, \quad z \in I,
$$

where $h:=h_{1} \circ T^{-1}$, so (4.1) holds.

Next, we replace the plane domain $D$ with a free analytic boundary arc by a bordered Riemann surface. Recall that a bordered Riemann surface $S$ with border $\Gamma$ is the disjoint union $S_{0} \cup \Gamma$ of the non-bordered Riemann surface $S_{0}=S \backslash \Gamma$ and its border $\Gamma$.

Theorem 4.3. Let $S=S_{0} \cup \Gamma$ be a bordered Riemann surface with border $\Gamma$, let $R$ be a Riemann surface, which carries a complete real analytic conformal metric $\lambda(w)|d w|$, let $h: \Gamma \rightarrow \mathbb{C}$ be a holomorphic function, and let $\mu$ be a positive and real analytic function on a neighborhood of $h(\Gamma)$. Then an analytic map $f: S_{0} \rightarrow R$ can be continued analytically across any compact subset $J$ of $\Gamma$ with $f(J) \subset R$, if

$$
\lim _{z \rightarrow \xi} \frac{\lambda(f(z))\left|f^{\prime}(z)\right||d z|}{\mu(h(z))\left|h^{\prime}(z)\right||d z|}=1, \quad \xi \in \Gamma .
$$

Remark 4.4. If $S$ is a bordered Riemann surface with border $\Gamma$, then a function $h: \Gamma \rightarrow \mathbb{C}$ is called holomorphic if it is defined and holomorphic in a neighborhood $U \subseteq S \cup S^{*}$ of $\Gamma$, where $S^{*}$ is the Schottky double of $S$. We say an analytic map $f$ from $S$ into a Riemann surface $R$ can be continued analytically across a subset $J \subseteq \Gamma$, if there exists a neighborhood $U \subseteq S \cup S^{*}$ of $J$ and an analytic map $F: U \rightarrow R$ such that $F$ agrees with $f$ in $U \cap S_{0}$.

Note that in Theorem 4.3 there exists by assumption an open subset $U$ of the double $S \cup S^{*}$ of $S$ such that $h$ is defined and holomorphic on $U$ and $\mu$ is defined on $h(U)$. Thus $\mu(h(z))\left|h^{\prime}(z)\right||d z|$ is the pullback of the conformal metric $\mu(\zeta)|d \zeta|$ on $h(U) \subset \mathbb{C}$ to the Riemann surface $S_{0} \cap U$ by the map $\left.h\right|_{S_{0} \cap U}$ and is therefore a 
conformal pseudo-metric on $S_{0} \cap U$. Also, $\lambda(f(z))\left|f^{\prime}(z)\right||d z|$ is just the pullback of the conformal metric $\lambda(w)|d w|$ by the analytic map $f: S_{0} \rightarrow R$, and hence a conformal pseudo-metric on $S_{0} \cap U$. Consequently (see Section 2.1), the quotient

$$
\frac{\lambda(f(z))\left|f^{\prime}(z)\right||d z|}{\mu(h(z))\left|h^{\prime}(z)\right||d z|}
$$

is a well-defined function from $S_{0} \cap U$ to $[0, \infty]$.

Proof. Let $\xi_{0}$ be a point on the border $\Gamma$. It suffices to show there is a neighborhood $W \subseteq S \cup S^{*}$ of $\xi_{0}$ and an analytic continuation $F$ of $f$ to $W$. Let $Y$ be the universal covering surface of $S \cup S^{*}$ and let $\pi: Y \rightarrow S \cup S^{*}$ be an analytic universal cover projection. Choose a point $z_{0} \in Y$ over $\xi_{0}$, let $U \subseteq S \cup S^{*}$ be a neighborhood of $\xi_{0}$, and let $V \subseteq Y$ be a neighborhood of $z_{0}$ such that $\pi$ is a conformal map from $V$ onto $U$. We may assume $V \subset \mathbb{C}$. Now, let $U_{\alpha} \cup U_{\alpha}^{*}$ be a parameter neighborhood of $\xi_{0}$ and let $\hat{\varphi}_{\alpha}$ be a local parameter on $U_{\alpha} \cup U_{\alpha}^{*}$. We may choose $U$ so small that $U \subset U_{\alpha} \cup U_{\alpha}^{*}, h$ is defined and holomorphic in $U$, and $\hat{\varphi}_{\alpha}$ maps $U$ onto the disk $K_{r}(0)$ with $\hat{\varphi}_{\alpha}\left(\xi_{0}\right)=0$ and $\hat{\varphi}_{\alpha}^{-1}\left(K_{r}^{+}(0)\right) \subset S$ and $\hat{\varphi}_{\alpha}^{-1}\left(K_{r}^{-}(0)\right) \subset S^{*}$. Then $\Psi:=\pi^{-1} \circ \hat{\varphi}_{\alpha}^{-1}$ maps $K_{r}(0)$ conformally onto $V$ and $I:=\Psi((-r, r))$ is a free analytic boundary arc of the domain $D:=\Psi\left(K_{r}^{+}(0)\right)$. Finally, let $g:=f \circ \pi: D \rightarrow R$ and observe that for $z=\pi(v)$,

$$
\lim _{v \rightarrow v_{0}} \frac{\lambda(g(v))\left|g^{\prime}(v)\right|}{\mu(h(\pi(v)))\left|(h \circ \pi)^{\prime}(v)\right|}=\lim _{z \rightarrow \xi} \frac{\lambda(f(z))\left|f^{\prime}(z)\right|}{\mu(h(z))\left|h^{\prime}(z)\right|}=1, \quad v_{0} \in I .
$$

By Theorem 4.1, $g$ has an analytic extension $G$ to a disk $K_{\rho}\left(z_{0}\right) \subset V \subset Y$. Therefore, $F=G \circ \pi^{-1}$ is an analytic extension of $f$ to the neighborhood $W:=$ $\pi\left(K_{\rho}\left(z_{0}\right)\right) \subseteq S \cup S^{*}$ of $\xi_{0}$.

Finally, we replace the holomorphic function $h: \Gamma \rightarrow \mathbb{C}$ in Theorem 4.3 by an analytic map $h$ from $\Gamma$ into a Riemann surface $Q$.

Theorem 4.5. Let $S=S_{0} \cup \Gamma$ be a bordered Riemann surface with border $\Gamma$, let $R$ be a Riemann surface with a complete real analytic conformal metric $\lambda(w)|d w|$, and let $Q$ be a Riemann surface with a real analytic conformal metric $\mu(\zeta)|d \zeta|$. Also, let $h: \Gamma \rightarrow Q$ be an analytic map. Then an analytic map $f: S \rightarrow R$ can be continued analytically across any compact subset $J$ of $\Gamma$ with $f(J) \subset R$, if

$$
\lim _{z \rightarrow \xi} \frac{\lambda(f(z))\left|f^{\prime}(z)\right|}{\mu(h(z))\left|h^{\prime}(z)\right|}=1, \quad \xi \in \Gamma .
$$

Proof. Let $\xi_{0} \in \Gamma$ and let $U \subset S \cup S^{*}$ be a simply connected neighborhood of $\xi_{0}$ such that $h$ is defined and analytic in $U$. Also, let $X$ be the universal covering surface of $Q$ and let $\tau: X \rightarrow Q$ be an analytic universal cover projection. Choose a point $\eta_{0} \in X$ over $h\left(\xi_{0}\right)$ and note that we may assume $\eta_{0} \neq \infty$. Then

$$
\left(\tau^{*} \mu\right)(\eta)|d \eta|=\mu(\tau(\eta))\left|\tau^{\prime}(\eta)\right||d \eta|
$$

is a real analytic conformal metric on $X$, and $h$ lifts to an analytic map $k: U \rightarrow X$ with $\tau \circ k=h$ in $U$ and $k\left(\xi_{0}\right)=\eta_{0} \neq \infty$. Shrinking $U$, if necessary, we may assume $k: U \rightarrow \mathbb{C}$ is a holomorphic function. Then

$$
\lim _{z \rightarrow \xi} \frac{\lambda(f(z))\left|f^{\prime}(z)\right|}{\left(\tau^{*} \mu\right)(k(z))\left|k^{\prime}(z)\right|}=\lim _{z \rightarrow \xi} \frac{\lambda(f(z))\left|f^{\prime}(z)\right|}{\mu(h(z))\left|h^{\prime}(z)\right|}=1, \quad \xi \in \Gamma \cap U
$$

so Theorem 4.3 implies that $f$ has an analytic continuation $F: W \rightarrow R$ to a neighborhood $W \subset U$ of $\xi_{0}$. 
4.2. The continuation process in euclidean, hyperbolic and spherical geometry. In this final section we take a closer look at the actual process of analytic continuation used in the proof of Theorem 1.1. For simplicity, we restrict ourselves to the situation of the 'if' part of Theorem $\mathrm{A}$, Theorem $\mathrm{B}$ and Theorem 1.8 , respectively, with $h(z) \equiv z$.

Let $k \in\{-4,0,+4\}$ and let $f$ be a holomorphic (or meromorphic) function in $\mathbb{D}$ satisfying

$$
\lim _{z \rightarrow \xi} \frac{\left|f^{\prime}(z)\right|}{1+\frac{k}{4}|f(z)|^{2}}=1, \quad \xi \in I,
$$

for some open subarc $I \subseteq \partial \mathbb{D}$. Put $J:=\partial \mathbb{D} \backslash I$. The proof in Section 3.1 reveals that the holomorphic (or meromorphic) continuation $F(z)$ of $f(z)$ to a neighborhood $U$ of $I$ is given by

$$
F(z)= \begin{cases}f(z), & z \in \mathbb{D}, \\ \frac{g\left(\frac{1}{z}\right)}{}, & z \in(\mathbb{C} \backslash \mathbb{D}) \cap U,\end{cases}
$$

where $g(z), z \in \mathbb{D} \cap U$, is the solution of the complex differential equation

$$
\begin{aligned}
g^{\prime}(z) & =-\frac{1}{z^{2} f^{\prime}(z)}\left(1+\frac{k}{4} f(z) g(z)\right)^{2} \\
& =-\frac{1}{z^{2} f^{\prime}(z)}-\frac{k f(z)}{2 z^{2} f^{\prime}(z)} g(z)-\frac{k^{2} f(z)^{2}}{16 z^{2} f^{\prime}(z)} g(z)^{2}, \quad z \in \mathbb{D},
\end{aligned}
$$

with $g(z)=\overline{f(1 / \bar{z})}$ for $z \in I$. Note that (4.2) is a complex Riccati differential equation for $g(z)$ with meromorphic coefficient functions in $\mathbb{D}$.

In the euclidean case $(k=0)$, this Riccati equation is particularly simple and takes the form

$$
g^{\prime}(z)=-\frac{1}{z^{2} f^{\prime}(z)}, \quad z \in \mathbb{D}
$$

Thus $g$ is obtained by an elementary integration. Clearly, $g$ can be continued holomorphically along every path in $\mathbb{D}$ that does not meet the origin or any critical point of $f(z)$, i.e. a point $z \in \mathbb{D}$ with $f^{\prime}(z)=0$. Thus $f$ has a holomorphic extension to any simply connected domain $\Omega$ with $\mathbb{D} \subseteq \Omega \subseteq \mathbb{C} \backslash J$, which contains no reflected critical point of $f$, i.e. if $f^{\prime}(z)=0$ for some $z \in \mathbb{D}$, then $1 / \bar{z} \notin \Omega$.

If $k=-4$, the hyperbolic case, then (4.2) is a real Riccati equation and it is clear from the general theory of such equations [14 that $g$ can be continued meromorphically along every path in $\mathbb{D}$, which avoids the origin and the critical points of $f$. In other words, the holomorphic function $f: \mathbb{D} \rightarrow \mathbb{D}$ has a meromorphic extension to any simply connected domain $\Omega$ with $\mathbb{D} \subseteq \Omega \subseteq \mathbb{C} \backslash J$, which contains none of the reflected critical points of $f$. One might suspect that $f$ even has a holomorphic continuation to such domains. In the language of complex differential equations this would mean that no movable singularities 5 can occur. But this is still an open problem. Note the structure of the local solutions of (4.2) is already pretty complicated; see [12].

Finally, in the spherical situation $(k=+4)$, the general theory of Riccati equations tells us that the meromorphic function $f: \mathbb{D} \rightarrow \mathbb{P}$ has a meromorphic continuation to any simply connected domain $\Omega$ with $\mathbb{D} \subseteq \Omega \subseteq \mathbb{C} \backslash J$, which contains none of the reflected critical points and none of the reflected poles of $f$.

${ }^{5}$ bewegliche Singularitäten 


\section{ACKNOWLEDGEMENTS}

The author thanks D. Kraus, Ch. Pommerenke, St. Ruscheweyh and E. Schippers for many useful discussions and suggestions. Special thanks are due to Ch. Pommerenke who suggested a vast simplification of the proof of Lemma 2.4

\section{REFERENCES}

[1] L. V. Ahlfors and L. Sario, Riemann surfaces, Princeton University Press, Princeton, 1960. MR0114911 (22:5729)

[2] F. G. Avkhadiev, Conformal mappings that satisfy the boundary condition of equality of metrics, Doklady Akad. Nauk. (1996), 347 no. 3, 295-297. English transl.: Doklady Mathematics. (1996), 53 no. 2, 194-196. MR1393054 (97d:30007)

[3] A. Beurling, An extension of the Riemann mapping theorem, Acta Math. (1953), 90, 117-130. MR0060027 (15:614e)

[4] C. Carathéodory, Zum Schwarzschen Spiegelungsprinzip (Die Randwerte von meromorphen Funktionen), Comment. Math. Helv. (1946), 46, 263-278. MR0020144 (8:508m)

[5] E. A. Coddington and N. Levinson, Theory of ordinary differential equations, McGraw-Hill, New York, 1955. MR0069338(16:1022b)

[6] P. L. Duren, Univalent Functions, Springer-Verlag, Berlin, New York, 1983. MR0708494 (85j:30034)

[7] R. Fournier and St. Ruscheweyh, Free boundary value problems for analytic functions in the closed unit disk, Proc. Amer. Math. Soc. (1999), 127 no. 11, 3287-3294. MR1618666 (2000b:30059)

[8] R. Fournier and St. Ruscheweyh, A generalization of the Schwarz-Carathéodory reflection principle and spaces of pseudo-metrics, Math. Proc. Cambridge Phil. Soc. (2001), 130, 353364. MR,1806784 (2003a:30022)

[9] F. W. Gehring, Characteristic properties of quasidisks, Les Presses de l'Université de Montréal, Montreal, Que., 1982. MR0674294 (84a:30036)

[10] M. Gromov, Metric Structures for Riemannian and non-Riemannian Spaces, Birkhäuser, Boston, 1999. MR1699320 (2000d:53065)

[11] J. Jost, Compact Riemann Surfaces: An Introduction to Contemporary Mathematics, Springer-Verlag, Berlin, New York, 1991. MR.1632873 (2000k:32011)

[12] D. Kraus, Riccati-Differentialgleichungen im Komplexen, Diploma Thesis, Universität Würzburg, 2000.

[13] R. Kühnau, Längentreue Randverzerrung bei analytischer Abbildung in hyperbolischer und sphärischer Geometrie, Mitt. Math. Sem. Giessen (1997), 229, 45-53. MR1439207 (98g:30011)

[14] I. Laine, Nevanlinna Theory and Complex Differential Equations, de Gruyter, Berlin, New York, 1993. MR1207139(94d:34008)

[15] J. Liouville, Sur l'équation aux différences partielles $\frac{d^{2} \log \lambda}{d u d v} \pm \frac{\lambda}{2 a^{2}}=0$, J. de Math. (1853), 16, 71-72.

[16] D. Minda, Conformal Metrics (unpublished).

[17] D. Minda, Bloch constants, J. Anal. Math. (1982), 41, 54-84. MR0687945 (85e:30013)

[18] Ch. Pommerenke, Boundary behaviour of conformal maps, Springer-Verlag, Berlin, New York, 1992. MR.1217706 (95b:30008)

[19] O. Roth, An extension of the Schwarz-Carathéodory reflection principle, Habilitationsschrift, Universität Würzburg, 2003.

[20] H. A. Schwarz, Über einige Abbildungsaufgaben, J. Reine Angew. Math. (1869), 70, 105-120.

[21] G. Warnecke, Über einige Probleme bei einer nichtlinearen Differentialgleichung zweiter Ordnung im Komplexen, J. Reine Angew. Math. (1969), 239-240, 353-362. MR0255955 (41:615)

[22] H. Whitney, Analytic extensions of differentiable functions defined in closed sets, Trans. Amer. Math. Soc. (1936), 36, 63-89. MR1501735

Mathematisches Institut, Universität Würzburg, D-97074 Würzburg, Germany

E-mail address: roth@mathematik.uni-wuerzburg.de 